NASA

Technical c. 1

Paper

2098

Reduction Methods for

Nonlinear Steady-State

Thermal Analysis

Ahmed K. Noor,

Chad D. Balch,

and Macon A. Shibut 


\title{
Reduction Methods for Nonlinear Steady-State Thermal Analysis
}

\author{
Ahmed K. Noor, \\ Chad D. Balch, \\ and Macon A. Shibut \\ The George Washington University \\ Joint Institute for Advancement of Flight Sciences \\ Langley Research Center \\ Hampton, Virginia
}


SUMMARY . . . . . . . . . . . . . . . . . . . . . . . . . . . . 1

INTRODUCTION . . . . . . . . . . . . . . . . . . . . . . . . 1

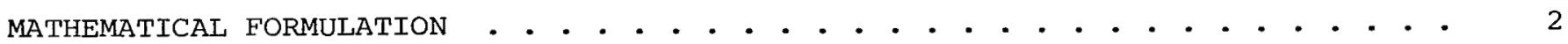

Governing Finite-Element Equations . . . . . . . . . . . . . . . . . . . 2

Solution of the Governing Equations . . . . . . . . . . . . . . . . . . . 3

BASIC IDEA OF REDUCTION METHODS ............................ . . . . . . . 4

Basis Reduction and the Reduced System of Equations . . . . . . . . . . . . . 4

Selection of Basis Vectors . . . . . . . . . . . . . . . . . . . 5

Comparison With Taylor Series Expansion . . . . . . . . . . . . . . . . 6

Comments on the selection of Basis Vectors . . . . . . . . . . . . . . . 7

COMPUTATIONAL ALGORITHM USED WITH REDUCTION METHODS . . . . . . . . . . . . . . 7

Evaluation of the Basis Vectors and Generation of the Reduced System

of Equations . . . . . . . . . . . . . . . . . . . . . . . 8

Sensing and Controlling the Error in the Reduced System of Equations . . . 8

NUMERICAL STUDIES . . . . . . . . . . . . . . . . . . . . . . . . 9

Two-Dimensional Steady-State Conduction in a Square Plate . . . . . . . . . 9

Two-Dimensional Steady-State Conduction in a Cylindex with an

Eccentric Hole .. . . . . . . . . . . . . . . . . . 10

Steady-State Analysis of a One-Dimensional Conducting-

Convecting-Radiating Fin . . . . . . . . . . . . . . . . . . . . . 11

Steady-State Conduction and Radiation in a segment of the Space

Shuttle Orbiter wing ... . . . . . . . . . . . . . . . . . 11

COMMENTS ON REDUCTION METHODS FOR NONLINEAR THERMAL ANALYSIS . . . . . . . • $\quad 12$

CONCLUDING REMARKS . . . . . . . . . . . . . . . . . . . . . . . 13

APPENDIX A - FORMULAS FOR COEFFICIENTS IN THE FINITE-ELEMENT EQUATIONS

FOR INDIVIDUAL ELEMENTS . . . . . . . . . . . . . . . . . . . . 15

APPENDIX B - EXPLICIT FORMS OF THE REDUCED SYSTEM OF EQUATIONS . . . . . • . . 17

APPENDIX C - EVALUATION OF THE PATh DERIVATIVES . . . . . . . . . . . . . . 19

APPENDIX D - TREATMENT OF PRESCRIBED BOUNDARY TEMPERATURES . . . . . . . . • . 26

APPENDIX E - EXACT SOLUTION FOR TWO-DIMENSIONAL STEADY-STATE CONDUCTION

IN A SQUARE PLATE WITH TEMPERATURE-DEPENDENT THERMAL CONDUCTIVITIES . . . . . 28

REFERENCES . . . . . . . . . . . . . . . . . . . . . . . . . 29

SYMBOLS . . . . . • . . . . . . . . . . . . . . . . . . . . . . 30

FIGURES . . . . . . . . . . . . . . . . . . . . . . . . . . . 33 


\section{SUMMARY}

Hybrid analysis techniques based on the combined use of finite elements and the classical Bubnov-Galerkin approximation are presented for predicting nonlinear steady-state temperature distributions in structures and solids. In these hybrid techniques the modeling versatility of the finite-element method is preserved and a substantial reduction in the number of degrees of freedom is achieved by expressing the vector of nodal temperatures as a linear combination of a small number of globaltemperature modes, or basis vectors. The Bubnov-Galerkin technique is then used to compute the coefficients of the linear combination (i.e., the amplitudes of the global-temperature modes).

The basis vectors chosen are the path derivatives commonly used in perturbation techniques, namely, the derivatives of the nodal-temperature vector with respect to a preselected control (or path) parameter or parameters. The vectors are generated by using the finite-element model of the initial discretization. Also, the performance of alternate sets of basis vectors is investigated. In the alternate sets, only a few path derivatives are generated, and they are augmented by a constant vector representing a uniform temperature rise (or drop) and by reciprocal vectors with nonzero components equal to the reciprocals of the nonzero components of the path derivatives. A problem-adaptive computational algorithm is presented for efficient evaluation of global approximation vectors and generation of the reduced system of equations and for monitoring the accuracy of the reduced system of equations.

The potential of the proposed reduction methods for the solution of large-scale, nonlinear steady-state thermal problems is also discussed. The effectiveness of these methods is demonstrated by means of four numerical examples, including conduction, convection, and radiation modes of heat transfer.

This study shows that the use of the uniform-temperature mode and the path derivatives as global approximation vectors significantly increases the accuracy of the solutions obtained by reduction methods, thereby enhancing the effectiveness of these methods for the solution of large-scale, nonlinear thermal problems.

\section{INTRODUCTION}

Computational methods for nonlinear heat transfer have recently become the focus of intense research efforts because of the need for realistic modeling and accurate thermal analysis of large, complex hardware systems subject to harsh environments (e.g., reentry flight vehicle structures, large-area space structures, and nuclear reactor components). Considerable progress has been made in the development of numerical discretization techniques. (See, for example, refs. 1 to 3.) Also, a number of versatile and powerful finite-element and finite-difference (lumpedparameter) programs have evolved for nonlinear thermal analysis. A survey of some of these programs is given in reference 4. In spite of these advances, the nonlinear thermal analyses of most large and complex hardware systems require excessive amounts of computer time even on present-day large computers and thus are very expensive.

The large numbers of degrees of freedom required in the nonlinear thermal analysis of complex systems are often dictated by the topology of the system rather than 
by the expected complexity of the behavior. A similar situation has been observed in nonlinear structural analysis problerns, and an effective reduction technique has been developed to significantly reduce the computational effort. (See refs. 5 and 6. ) The application of the reduction method of reference 6 to transient nonlinear thermal problems is described in reference 7. The aim of the present study is to develop a reduction method and a computational algorithm for nonlinear steady-state thermal analysis of structures and solids. The proposed technique is similar to that presented in references 5 and 6 for the nonlinear static analysis of structures and is a hybrid method which combines the modeling versatility of contemporary finite elements with the reduction in the total number of degrees of freedom provided by the classical Bubnov-Galerkin technique.

To sharpen the focus of the study, discussion is limited to nonlinear steadystate thermal problems with continuous temperature fields in the space domain. Convection, nonlinear conduction, and radiation modes of heat transfer are considered.

\section{MATHEMATICAL FORMULATION}

\section{Governing Finite-Element Equations}

The solid region is discretized by using a single-field finite-element model with the fundamental unknowns consisting of the temperatures at the various nodes. The governing finite-element thermal-equilibrium equations can be cast in the following form:

$$
\{f(T)\}=[K(T)]\{T\}-q\{Q\}=0
$$

where $\{\mathrm{T}\}$ is the vector of nodal temperatures, $\{Q\}$ is the normalized thermalload vector, $[\mathrm{K}(\mathrm{T})]$ is the $\mathrm{n} \times \mathrm{n}$ heat-transfer matrix ( $\mathrm{n}$ is the total number of degrees of freedom), and $q$ is a normalizing parameter for the thermal-load vector. (A list of symbols used in this paper appears after the references.) The matrix $[\mathrm{K}(\mathrm{T})]$ and the vector $\{Q\}$ can each be decomposed into three components as follows:

$$
[K(T)]=\left[K^{(k)}\right]+\left[\bar{K}^{(K)}\right]+\left[K^{(h)}\right]
$$

and

$$
\{Q\}=\left\{Q^{(0)}\right\}+\{Q(r)\}+\{Q(h)\}
$$

where $\left[\mathrm{K}^{(\mathrm{K})}\right],\left[\overline{\mathrm{K}}^{(\mathrm{r})}\right]$, and $\left[\mathrm{K}^{(\mathrm{h})}\right]$ are the conduction, radiation, and convection matrices; and $\left\{Q^{(O)}\right\},\left\{Q^{(r)}\right\}$, and $\{Q(h)\}$ are normalized applied heating, radiation, and convection thermal-load vectors. For convenience, the material conductivities in the present study are assumed to vary quadratically with temperature, as follows :

$$
k_{\alpha \beta}=k_{\alpha \beta}^{\circ}\left(1+\gamma_{1} T+\gamma_{2} T^{2}\right)
$$


where $k_{\alpha \beta}^{0}$ is the thermal-conductivity coefficient at a preselected reference temperature of $T=0$ and $\gamma_{1}$ and $\gamma_{2}$ are conductivity coefficients. In the numerical studies, only isotropic materials are considered, for which $k_{11}=k_{22}=k^{\circ}\left(1+\gamma_{1} \mathrm{~T}+\gamma_{2} \mathrm{~T}^{2}\right)$ and $k_{12}=0$. Also, the convection matrix is assumed to be independent of temperature and the radiation matrix varies cubically with temperature.

The matrices $\left[\mathrm{K}^{(\mathrm{k})}\right]$ and $\left[\overline{\mathrm{K}}^{(\mathrm{r})}\right]$ can be expressed in the following forms:

$$
\left[K^{(k)}\right]=\left[K^{(0)}\right]+q_{1}\left[K^{(1)}(T)\right]+q_{2}\left[K^{(2)}(T)\right]
$$

and

$$
\left[\bar{K}^{(x)}\right]=q_{3}\left[K^{(x)}\right]
$$

where $\left[\mathrm{K}^{(0)}\right]$ is the linear conduction matrix (independent of $\mathrm{T}$ ); [K(1)] and $\left[\mathrm{K}^{(2)}\right]$ are normalized nonlinear conduction matrices whose terms are linear and quadratic in the nodal temperatures $\{\mathrm{T}\} ;[\mathrm{K}(\mathrm{r})]$ is a normalized radiation matrix; and $q_{1}, q_{2}$, and $q_{3}$ are normalizing parameters. The expressions for $\left[\mathrm{K}^{(0)}\right]$, $\left[K^{(1)}\right],\left[K^{(2)}\right],\left[K^{(r)}\right],\left[K^{(h)}\right],\left\{Q^{(0)}\right\},\left\{Q^{(r)}\right\}$, and $\left\{Q^{(h)}\right\}$ for the individual elements are given in appendix $A$.

\section{Solution of the Governing Equations}

The solution of equation ( 1 ) is obtained by using an incremental-iterative technique (e.g., a predictor-corrector continuation method). This is accomplished by embedding equation ( 1 ) in a single- or multiple-parameter family of equations of the form $\{f(T, q)\}=0$ or $\left\{f\left(T, q_{1}, q_{2}, q_{3}\right)\right\}=0$. Only the single-parameter case is discussed in this section. The multiple-parameter case is examined in subsequent sections (in conjunction with reduction methods). The normalizing parameters $q$, $\mathrm{q}_{1}, \mathrm{q}_{2}$, and $\mathrm{q}_{3}$ are also referred to as control or path parameters. Henceforth, the terms normalizing parameter, control parameter, and path parameter are used interchangeably.

In the single-parameter case, $q_{1}=q_{2}=q_{3}=1$. For each value of $q$ in some interval (e.g., $0 \leq \mathrm{q} \leq \overline{\mathrm{q}}$ ), a solution $\{\mathrm{T}(\mathrm{q})\}$ exists which varies continuously with $q$ and satisfies the conditions $\{\mathrm{T}(0)\}=\{0\}$ and $\{\mathrm{T}(\overline{\mathrm{q}})\}=\{\overline{\mathrm{T}}\}$. The solution $\{\mathrm{T}\}$ corresponding to a particular value of the parameter $q$ is used to calculate a suitable approximation (predictor) for $\{\mathrm{T}\}$ at a different value of $q$. This approximation is then chosen as an initial estimate for $\{\mathrm{T}\}$ in a correctiveiterative scheme such as the Newton-Raphson technique. The iteration process is represented by the following equations for the $j$ th iteration cycle:

$$
[J]^{(j)}\{\Delta T\}^{(j)}=-\{f(T, q)\}(j)
$$


and

$$
\{\}^{(j+1)}=\{T\}^{(j)}+\{\Delta T\}^{(j)}
$$

where $[J]$ is the Jacobian matrix defined as

$$
[J]=[K]+\left[\frac{\partial K_{I L}}{\partial T_{J}} T_{L}\right]
$$

Note that the Jacobian matrix $[\mathrm{J}]$ is, in general, nonsymmetric. In the present study, in order to avoid the complications associated with solving nonsymmetric equations, a quasi-Newton method is used. The method is based on using a symmetric

approximation to the Jacobian matrix consisting of the sum of the matrix $\left[K^{(0)}\right]$ and the contribution of the radiation matrix to $\left[\frac{\partial \mathrm{K}_{I L}}{\partial \mathrm{T}_{J}} \mathrm{~T}_{L}\right]$. The contributions of the nonlinear conduction matrices to $\left[\frac{\partial \mathrm{K}_{I L}}{\partial \mathrm{T}_{J}} \mathrm{~T} L\right]$ form a nonsymmetric matrix and are neglected.

\section{BASIC IDEA OF REDUCTION METHODS}

\section{Basis Reduction and the Reduced System of Equations}

The essence of reduction methods is to reformulate the problem in terms of a few discrete variables $\{\psi\}$, which represent amplitudes of global-temperature modes (functions of the variables $\{T\}$ ). This is accomplished by using the following transformation:

$$
\{\mathrm{T}\}_{\mathrm{n}, 1}=[\mathrm{T}]_{\mathrm{n}, \mathrm{r}}\{\psi\}_{\mathrm{r}, \mathrm{l}}
$$$$
(x<<n)
$$

where $[\Gamma]$ is an $n \times r$ transformation matrix in which the columns represent global approximation (or basis) vectors. Note that the number of generalized discrete variables $r$ is assumed to be much smaller than the number of degrees of freedom of the initial discretization $n$.

A Bubnov-Galerkin technique is then used to approximate equation (1) with a much smaller system of nonlinear algebraic equations in the new unknowns $\{\psi\}$. The reduced equations have the following form:

$$
\{\tilde{f}(\psi)\}=[\tilde{K}(\psi)]\{\psi\}-q\{\tilde{Q}\}=0
$$

where

$$
[\tilde{K}(\psi)]=[\Gamma]^{T}[K(\psi)][\Gamma]
$$


and

$$
\{\tilde{Q}\}=[\Gamma]^{\mathrm{T}}\{Q\}
$$

The tilde $(\sim)$ refers to the reduced system, the superscript $T$ denotes transposition, and $[\mathrm{K}(\psi)]$ is obtained from $[\mathrm{K}(\mathrm{T})]$ by replacing $\{\mathrm{T}\}$ by its expression in terms of $\{\psi\}$ from equation (10).

The reduced-system heat-transfer matrix $[\tilde{\mathrm{K}}]$ and thermal-load vector $\{\tilde{Q}\}$ can be separated into components as follows (see eqs. (2), (3), (5), and (6)):

$$
[\tilde{\mathrm{K}}(\psi)]=\left[\tilde{\mathrm{K}}^{(0)}\right]+\mathrm{q}_{1}\left[\tilde{\mathrm{K}}^{(1)}\right]+\mathrm{q}_{2}\left[\widetilde{\mathrm{K}}^{(2)}\right]+\mathrm{q}_{3}\left[\widetilde{\mathrm{K}}^{(\mathrm{r})}\right]+\left[\tilde{\mathrm{K}}^{(\mathrm{h})}\right]
$$

and

$$
\{\tilde{Q}\}=\left\{\tilde{Q}^{(0)}\right\}+\left\{\tilde{Q}^{(r)}\right\}+\left\{\tilde{Q}^{(h)}\right\}
$$

where $\left[\tilde{\mathrm{K}}^{(0)}\right]$ is the linear conduction matrix; $\left[\tilde{\mathrm{K}}^{(1)}\right]$ and $\left[\tilde{\mathrm{K}}^{(2)}\right]$ are the normalized nonlinear conduction matrices; $\left[\tilde{\mathrm{K}}^{(h)}\right]$ and $\left[\tilde{\mathrm{K}}^{(r)}\right]$ are the convection and normalized radiation matrices; $\left\{\tilde{Q}^{(0)}\right\}$ is the normalized applied heating vector; and $\left\{\tilde{Q}^{(r)}\right\}$ and $\left\{\tilde{Q}^{(h)}\right\}$ are normalized radiation and convection thermal-load vectors. The explicit forms of the reduced-system arrays are given in appendix $B$.

The solution of the reduced-system nonlinear equations (eq. (11)) is obtained by using an incremental-iterative technique similar to the one described in the preceding section for the full system of equations. However, since the number of equations is small and their solution time is a small fraction of the total analysis time, no approximation needs to be made for the reduced-system Jacobian matrix [j̃], and the nonsymmetric contributions of the nonlinear conduction matrices to $\left[\frac{\partial \tilde{\mathrm{K}}_{i \ell}}{\partial \psi_{j}} \psi_{\ell}\right]$
are included in $[\tilde{J}]$.

\section{Selection of Basis Vectors}

The crux of reduction methods is the proper selection of the reduced basis vectors (columns of the matrix $[\Gamma]$ in eq. (10)). An ad hoc or intuitive choice may not lead to a satisfactory approximation. A large number of numerical experiments with structural and solid mechanics problems have demonstrated that, in the case of a single path parameter (such as q), the various-order derivatives of $\{\mathrm{T}\}$ with respect to $\mathrm{q}$ (path derivatives) provide an effective set of basis vectors. (See refs. 5 and 6.) In the present study, the effectiveness of using such basis vectors for thermal problems is investigated. The path derivatives are evaluated at $q=0 \quad\left(q_{1}=q_{2}=q_{3}=1\right)$ and are given by

$$
[\Gamma]=\left[\left\{\frac{\partial T}{\partial q}\right\}_{0},\left\{\frac{\partial^{2} T}{\partial q^{2}}\right\}_{0}, \ldots,\left\{\frac{\partial^{r} T}{\partial q^{r}}\right\}_{0}\right]
$$


In the case of multiple path parameters (such as $q_{1}, q_{2}$, and $q_{3}$ ), the basis vectors are chosen to be the various-order derivatives with respect to these path parameters evaluated at $\mathrm{q}_{1}=\mathrm{q}_{2}=\mathrm{q}_{3}=0 \quad(\mathrm{q}=1)$, that is,

$$
[\Gamma]=\left[\{T\}_{0},\left\{\frac{\partial T}{\partial q_{1}}\right\}_{0},\left\{\frac{\partial T}{\partial q_{2}}\right\}_{0},\left\{\frac{\partial T}{\partial q_{3}}\right\}_{0},\left\{\frac{\partial^{2} T}{\partial q_{1}^{2}}\right\}_{0},\left\{\frac{\partial^{2} T}{\partial q_{1} \partial q_{2}}\right\}_{0},\left\{\frac{\partial^{2} T}{\partial q_{1} \partial q_{3}}\right\}_{0}, \ldots\right]
$$

where $\{\mathbf{T}\}_{0}$ is the linear solution (zeroth-order derivative). The equations used in evaluating the basis vectors (path derivatives) are obtained by successive differentiations of the governing finite-element equations of the initial discretization in equation (1). The explicit forms of these equations are given in appendix $C$ for both the single-parameter and the multiple-parameter cases. The advantage of computing the path derivatives at zero values of the path parameters is that the left-hand-side matrix used in evaluating the path derivatives is independent of temperature. (See appendix C.)

The chosen set of basis vectors is the same as that commonly used in classical perturbation techniques. The basis vectors have the following properties:

1. They are linearly independent and span the space of solutions in the neighborhood of the point of their generation, and therefore they fully characterize the nonlinear solution in that neighborhood.

2. Their generation, using the finite-element model of the initial discretization, requires only one matrix factorization of the linear matrix $\left[\left[\mathrm{K}^{(\mathrm{O})}\right]+\left[\mathrm{K}^{(\mathrm{h})}\right]\right]$ (see appendix $\mathrm{C}$ ), and therefore it is computationally inexpensive.

3. They provide a direct measure of the sensitivity of the thermal response to changes in the control (path) parameters.

The first property is necessary for the convergence of the Bubnov-Galerkin approximation. The second property significantly enhances the efficiency of the reduction method and increases its effectiveness in solving large-scale nonlinear thermal problems. The implication of the third property is that by appropriate choice of the control parameters, sensitivity of the temperature distribution to changes in the thermal data of the medium (e.g., nonlinear conduction and radiation coefficients) can be obtained. Note that the sensitivity information is obtained at zero value(s) of the control parameter(s). If the sensitivity is required at other values of the parameter(s), approximate Taylor series expansion(s) may be used.

It should be noted that the use of path derivatives as basis vectors in nonlinear structural and solid mechanics problems provided highly accurate solutions within a large neighborhood of the point of evaluation of these derivatives (refs. 5 and 6 ).

\section{Comparison With Taylor Series Expansion}

If the reduction method outlined in the preceding section is contrasted with the Taylor series expansion used in classical perturbation techniques, the following 
can be noted. In both methods the vector of nodal temperatures $\{\mathrm{T}\}$ is approximated, over a range of values of the path parameter(s), by a linear combination of the various-order derivatives of $\{\mathrm{T}\}$ with respect to the path parameter(s). However, the coefficients $\{\psi\}$ of the linear combination in the Taylor series expansion are fixed and are equal to

$$
\Delta q, \frac{(\Delta q)^{2}}{2 !}, \frac{(\Delta q)^{3}}{3 !}, \cdots
$$

for the single-parameter case and

$$
1, \Delta q_{1}, \Delta q_{2}, \Delta q_{3}, \frac{\left(\Delta q_{1}\right)^{2}}{2 !}, \Delta q_{1} \Delta q_{2}, \Delta q_{1} \Delta q_{3}, \ldots
$$

for the three-parameter case. By contrast, the coefficients $\{\psi\}$ in the reduction technique are left as free parameters and are determined by using the Bubnov-Galerkin technique. Therefore, the reduction method can be thought of as either of the following:

1. A generalized Taylor series (or perturbation) approach with free parameters or coefficients $\{\psi\}$

2. A generalized Bubnov-Galerkin approach with the approximation (or basis) vectors generated by using a preselected finite-element model rather than chosen a priori

Numerical experiments have shown that the use of the free parameters in the generalized Taylor series technique leads to accurate solutions not only within the radius of convergence of the Taylor series but also well beyond it. (see refs. 5 and 6.)

\section{Comments on the Selection of Basis Vectors}

The computational effort required to generate the basis vectors can be reduced by generating only a few path derivatives (four or five) using equations (C1) or (C2) and then augmenting these derivatives with a constant vector with equal components representing a uniform temperature mode, and/or reciprocal vectors with nonzero components equal to the reciprocals of the nonzero components of the vectors in equations (Cl) or (C2). The performance of the augmented set of basis vectors is discussed in the section entitled "Numerical Studies."

\section{COMPUTATIONAI ALGORITHM USED WITH REDUCTION METHODS}

To realize the potential of reduction methods in large-scale, nonlinear thermal analysis, a problem-adaptive computational algorithm is needed which is both robust and efficient. The two key elements of the algorithm for nonlinear steady-state problems are the following:

1. Efficient evaluation of the basis vectors and generation of the reduced system of equations

2. Sensing and controlling the error in the reduced system of equations 
These elements have been discussed in references 5 and 6 in connection with structural and solid mechanics applications. The key features of the procedure are discussed hereinafter.

\section{Evaluation of the Basis Vectors and Generation of the Reduced System of Equations}

The particular choice of the basis vectors to be the various-order derivatives of the nodal-temperature vector $\{\mathrm{T}\}$ with respect to the path parameter(s) permits the generation of all the vectors with only one factorization of the matrix $\left[\left[\mathrm{K}^{(0)}\right]+\left[\mathrm{K}^{(\mathrm{h})}\right]\right]$. (See appendix C.) Therefore, the effort to generate the second and succeeding basis vectors reduces to that of evaluating the right-hand sides $R_{I}^{(a)}$ of equations (C1) or (C2) for the single-parameter or the multiple-parameter cases. The expressions of the right-hand sides grow in complexity for higher order derivatives, and their computation involves contractions of multidimensional arrays with the basis vectors. (See appendix C.) To improve the computational efficiency, the contracted arrays which are common to more than one right-hand side are formed once and stored for subsequent use.

Note that because of the recursive nature of the formulas for the path derivatives in both the single-parameter and the multiple-parameter cases, all the lower order derivatives must be evaluated before any subsequent derivatives can be computed. Once the entire set of path derivatives has been generated, it can be augmented with a constant vector, reciprocal vectors, or the vector $\{z\}$ (eq. (D2) in appendix D) for the case of prescribed nonzero nodal temperatures to form the matrix $[\Gamma]$.

The formation of the reduced arrays involves contraction of the full-system arrays with the basis vectors and appears to be the most time-consuming part of the solution process based on the reduction technique. The independent elements of the reduced arrays are generated once and stored for subsequent use, when the temperature distribution is required for different value(s) of the path parameter(s).

\section{Sensing and controlling the Error in the} Reduced System of Equations

To check the accuracy of the solution obtained with the reduced system of equations at any value of the path parameter, the approximate nodal temperatures are generated by using equation (10) with the vector $\{\psi\}$ obtained by solving the reduced equations in equation (11). Then the residual vector $\{\mathrm{R}\}$ of the original finite-element equations (eq. (1)) is computed as follows:

$$
\{R\}=[\dot{K}(T)]\{T\}-q\{Q\}
$$

In the present study, a weighted Euclidean norm of $\{R\}$ is used as an error measure e, namely

$$
e=\frac{1}{q} \sqrt{\{R\}^{T}\{R\} /\left(\{Q\}^{T}\{Q\}\right)}
$$


Numerical experiments have demonstrated that the error norm e is useful in assessing the overall accuracy of different reduction methods (based on different sets of basis vectors). However, the magnitude of this error norm was found to be problem dependent, and therefore it is not possible to prescribe a value for the error tolerance which is suitable for different classes of nonlinear thermal problems. An alternate way of assessing the accuracy of the reduced solution is to use the vector of nodal temperatures $\{T\}$ generated by the reduced system as a predictor in the full system, obtain a corrected estimate of $\{\mathrm{T}\}$ by performing a single iteration of the Newton-Raphson technique, and compare the predicted and corrected values of $\{\mathrm{T}\}$.

\section{NUMERICAI STUDIES}

To test and evaluate the effectiveness of the proposed reduction techniques, several nonlinear thermal problems were solved. For each problem, solutions based on the full system of equations of the finite-element model were compared with other numerical approximations and exact solutions, whenever available; then the solutions obtained by the reduction method were compared with the full-system solutions and with other numerical approximations. The results of four typical problems are discussed herein. The four problems are the following: (a) two-dimensional steady-state conduction in a square plate; (b) two-dimensional steady-state conduction in a cylinder with an eccentric hole; (c) steady-state analysis of a one-dimensional conductingconvecting-radiating $\mathrm{fin}$; and (d) steady-state conduction and radiation in a segment of the Space Shuttle orbiter wing. In all the problems considered, the thermal conductivity is assumed to be temperature dependent. In the first two problems only the single-parameter reduction method is used, and in the last two problems both the single-parameter and the multiple-parameter methods are applied.

\section{Two-Dimensional Steady-State Conduction in a Square Plate}

The first problem considered is that of steady-state thermal conduction in a thin square plate with prescribed boundary temperatures. (See fig. 1.) The thermal conductivities vary quadratically with temperature. The material and geometric characteristics of the plate are given in figure 1 . The problem was solved with a number of techniques, including an exact analytic technique based on the Kirchhoff transformation, finite elements, single-parameter reduction methods, and a perturbation technique based on Taylor series expansion of the temperature. The details of the exact analytic solution are given in appendix E. For the case of linear variation of conductivity coefficients with temperature, an exact analytic solution and a perturbation solution are presented in reference 8 .

Because of symmetry, only one-half of the plate was considered and was modeled by a grid of $5 \times 10$ elements with biquadratic Lagrangian interpolation functions for the temperature (a total of 190 temperature degrees of freedom (D.O.F.)). The finite-element solution was found to be in close agreement with the exact analytic solution. In both the single-parameter reduction method and the Taylor series expansion, the control parameter $q$ was taken to be the amplitude of the nonzero prescribed boundary temperatures. Contour plots for the temperatures at $q=300 \mathrm{~K}$, $600 \mathrm{~K}$, and $2400 \mathrm{~K}$ are shown in figure 1. As can be seen from figure 1, at higher values of $q$ a boundary layer (with steep temperature gradients) is formed near the edges where a temperature of $\mathrm{O} \mathrm{K}$ is prescribed. The basis vectors were evaluated at $q=0 \mathrm{~K}$, and they were obtained by solving a linear set of finite-element equations. (See appendix C.) 
Four sets of basis vectors are considered in the reduction method. The first two sets of basis vectors consist of the following: (a) the first eight derivatives with respect to the control parameter $q$; and (b) the first four derivatives with respect to $q$ and four reciprocal vectors (with nonzero components equal to the reciprocals of the nonzero components of the path derivatives). The last two sets of basis vectors are the same as the first two, except for the addition of a constant vector. Normalized contour plots for the first six path derivatives are shown in figure 2 .

The accuracy of the solutions obtained by using the Taylor series expansion and the reduction method is indicated in figures 3 and 4 . Also, figure 5 shows the variations of the error norm e (eq. (19)) and the root-mean-square error as the number of reduced-system basis vectors increases. An examination of the results shown in figures 3 to 5 reveals the following:

1. The Taylor series solution is considerably in error, even for the lowest value of $q(q=300 \mathrm{~K})$. (See $\mathrm{fig}$. 3.) The radius of convergence of the Taylor series was found to be in the neighborhood of $q=240 \mathrm{~K}$. By contrast, the solutions obtained by using the single-parameter reduction method with eight basis vectors were generally accurate, except near the boundary zone. (See fig. 4.)

2. The accuracy of the solutions obtained by using four derivatives and four reciprocal vectors is comparable to that obtained by using the eight derivatives. (See fig. 4.) However, the computational effort involved in generating the eight derivatives is considerably more than that required for generating the four derivatives and the reciprocal vectors.

3. The accuracy of the solutions obtained by using the reduction method was considerably improved when the eight basis vectors were augmented by a constant vector. This is true for both sets of basis vectors. (See fig. 4.)

4. The variation of the error norm $e$ is similar to that of the root-mean-square error (see fig. 5), and the solutions obtained with the reduction method converge to the full-system solution as the number of basis vectors increases.

Two-Dimensional steady-State Conduction in a Cylinder With an Eccentric Hole

The second problem is steady-state conduction in a cylinder with an eccentric hole. The temperatures of the inner and outer surfaces of the cylinder are prescribed to be $1000 \mathrm{~K}$ and $\mathrm{O} \mathrm{K}$. The finite-element model along with the material and geometric characteristics of the cylinder is shown in figure 6. A previous finite-element solution is presented in reference 9 for linear variation of the conductivity coefficients with temperature. Because of symmetry, only one-half of the cross section was modeled by a grid of 48 isoparametric, 9-noded elements (a total of 195 temperature degrees of freedom). Contour plots for the temperatures are shown in figure 6 for the two cases of $\gamma_{2}=0$ and $\gamma_{2}=0.005$, which correspond to linear and quadratic variation of thermal conductivity with temperature. A boundary layer (with steep temperature gradients) exists near the outer surface when $\gamma_{2}=0.005$, as shown in figure 6. The control parameter $q$ was taken to be the amplitude of the prescribed boundary temperature. The basis vectors were generated at $q=0$. 
Four sets of basis vectors are again considered, namely, eight derivatives with respect to the control parameter $\mathrm{q}$, eight derivatives and a constant vector, four derivatives with respect to $\mathrm{q}$ and their reciprocal vectors, and four derivatives, their reciprocal vectors, and a constant vector. The accuracy of the solutions obtained by using each of the sets of basis vectors is indicated in figure 7 for the two cases of $\gamma_{2}=0$ and $\gamma_{2}=0.005$. As in the previous problem, the addition of the constant vector considerably improves the accuracy of the solutions, particularly near the outer surface for the case of $\gamma_{2}=0.005$. (Note that the error in the solution obtained without the use of the constant vector is represented by the distance between the cross and circle symbols in fig. 7.) Also, the accuracy of the solutions obtained by using four derivatives and four reciprocal vectors is comparable to that obtained by using the eight derivatives.

\author{
Steady-State Analysis of a One-Dimensional \\ Conducting-Convecting-Radiating $\mathrm{F}$ in
}

The third problem considered is steady-state analysis of a one-dimensional conducting-convecting-radiating fin. (See fig. 8.) The tempexature at one end is prescribed to be $1000 \mathrm{~K}$. The fin was modeled by using a variable grid of 15 onedimensional finite elements having 3 nodes and quadratic Lagrangian interpolation functions for the temperature (a total of 30 temperature degrees of freedom). The thermal conductivity is assumed to vary linearly with temperature, and therefore the nonlinear conduction matrix $\left[\mathrm{K}^{(2)}(\mathrm{T})\right]$ is zero.

Solutions were obtained by using both the single-parametex and the two-parameter reduction methods. In the single-parameter method, the control parameter $q$ is the magnitude of both the prescribed temperature at $\mathrm{x}_{1}=0$ and the convection thermalload vector. The first six derivatives with respect to $q$ were generated at $q=0$. In the two-parameter method, the control parameters are chosen to be $q_{1}$ and $q_{3}$. Six path derivatives were generated at $q_{1}=q_{3}=0$. (These include the linear solution and all the first and second derivatives with respect to $q_{1}$ and $q_{3}$.) The accuracy of the solutions obtained by using the reduction methods is indicated in figure 9. The solutions obtained by using both the single-parameter and the twoparameter reduction methods are in close agreement with the full-system solution except near the edge where $\mathrm{x}_{1}=1.0$. The maximum errors in the temperature at $\mathrm{x}_{1}=1.0$ obtained with the single-parameter and the two-parameter reduction methods are 6.9 and 5.8 percent. In the presence of convection, it was necessary to add the constant vector to the two sets of basis vectors to obtain accurate solutions.

\title{
Steady-State Conduction and Radiation in a segment of the Space Shuttle orbiter wing
}

The fourth problem is steady-state conduction and radiation in a segment of the Space Shuttle oxbiter wing (fig. 10). The problem was selected to assess the effectiveness of reduction methods for complex aerospace applications.

The model consists of a two-dimensional cross section of a single bay of the Shuttle orbiter wing including reusable surface insulation (RSI) and RSI coating, room temperature-vulcanizing (RTV) rubber, a strain isolation pad (SIP), and part of the aluminum wing box structure. This model is an adaptation of a finite-element model developed by the authors of reference 10. The applied heating on the structure consists of a distributed heat source on the outer surface $Q\left(x_{1}\right)$, which varies 
quadratically with $x_{1}$. The temperature of the aluminum inner surface is prescribed as $394.4 \mathrm{~K}$ and the outer surface is a radiating boundary. The material and geometric characteristics of the structure and the finite-element model used are shown in figure 10. Note that the scale of the model is magnified in the $x_{2}$ direction for visual clarity. Rectangular nine-noded Lagrangian elements with biquadratic interpolation functions for the temperature are used in modeling the RSI, RTV, and SIP. The aluminum structure, RSI coating, and radiating boundary are modeled by using onedimensional three-noded elements with quadratic temperature interpolation functions. The finite-element model has a total of 64 nine-noded rectangular elements, 16 threenoded conduction elements, and 8 three-noded radiation elements (a total of 272 temperature degrees of freedom).

Solutions were obtained by using both the single-parameter and the threeparameter reduction methods. In the single-parameter method, the control parameter $q$ was the magnitude of both the prescribed temperature and the applied heating. A total of eight path derivatives were generated at $q=0$. In the three-parameter case, 10 path derivatives were generated at $q_{1}=q_{2}=q_{3}=0$.

The following two sets of basis vectors were used: the first eight derivatives with respect to the control parameter, and the linear solution and all the first- and second-order derivatives with respect to the control parameters $q_{1}$, $q_{2}$, and $q_{3}$. $A$ constant vector was added to each of the two sets. The accuracy of the solutions obtained by using each of the two sets of basis vectors is indicated in figure 11 , in which plots of the temperature distribution along Iines parallel to the $x_{1}$ and $x_{2}$ axes are shown. The variations of the error norm e and the root-mean-square error with the number of basis vectors are shown in figure 12. The addition of a constant vector to the path derivatives was necessary to obtain acceptable accuracy. (Results obtained without the use of the constant vector are not shown.)

An examination of the results shown in figures 11 and 12 reveals the following:

1. Both the single-parameter and the three-parameter reduction methods give accurate results for the high temperatures at the outer surface. However, the solutions obtained by using the single-parameter method are more accurate than those obtained by using the three-parameter method in the low-temperature region (near the aluminum structure).

2. The variation of the error norm e is similar to that of the root-mean-square error, and the solutions obtained with both the single-parameter and the threeparameter reduction methods converge to the full-system solution as the number of basis vectors increases.

\section{COMMENTS ON REDUCTION METHODS FOR NONLINEAR THERMAL ANALYSIS}

The proposed reduction methods appear to have high potential for solution of large-scale, nonlinear thermal problems. The numerical studies conducted clearly demonstrate the accuracy and effectiveness of the techniques. In particular, the following two points are worth mentioning:

1. The small size of the reduced system of equations makes it feasible to conduct parametric studies by repeatedly solving the equations for varying values of the control parameter(s). 
2. For the problems considered, the reduction in the analysis time achieved by using the proposed technique in general was not proportional to the reduction in the number of degrees of freedom of the initial discretization. This was due to the relatively high cost of evaluating the basis vectors and of generating the reduced equations (as compared with the cost of solving the reduced nonlinear algebraic equations). For any given problem, the saving in the analysis time resulting from the use of reduction methods is a function of the hardware and of the system software as well as the efficiency of the program used in the analysis, and therefore it is difficult to quantify. In the numerical examples considered, overall reductions in the CPU times are estimated to be factors of 2 to 3 . However, it should be noted that the program used for the computation was not optimized, and additional information in the form of sensitivity of the thermal response was provided by the basis vectors used in the proposed technique. More dramatic savings in CPU times are expected in largescale problems with thousands of degrees of freedom if an efficient computer implementation of the proposed technique is made.

\section{CONCLUDING REMARKS}

Reduction methods and a problem-adaptive computational algorithm were presented for predicting nonlinear steady-state temperature distributions in structures and solids. The computational algorithm can be conveniently divided into the following two distinct stages: discretization by using the finite-element method, and reduction in the number of degrees of freedom of the initial discretization by expressing the vector of unknown nodal temperatures as a linear combination of global-temperature modes, or basis vectors. A Bubnov-Galerkin technique is used to compute the coefficients of the linear combination (amplitudes of the global-temperature modes). The basis vectors are chosen to be those commonly used in the perturbation technique, namely, the derivatives of the nodal-temperature vector with respect to preselected control (or path) parameters. The vectors are generated by using the finite-element model of the initial discretization.

Four numerical examples demonstrate the effectiveness of reduction methods for the solution of nonlinear steady-state thermal problems. The four problems are twodimensional steady-state conduction in a square plate, two-dimensional steady-state conduction in a cylinder with an eccentric hole, steady-state analysis of a onedimensional conducting-convecting-radiating fin, and steady-state conduction and radiation in a segment of the Space shuttle orbiter wing. In all the problems considered, the thermal conductivity is assumed to be temperature dependent.

The results of the study suggest several conclusions relative to the selection of basis vectors and to the effectiveness of using reduction methods in nonlinear steady-state thermal problems. These conclusions are as follows:

1. The proposed reduction methods are hybrid techniques which combine the major advantages of the finite-element numerical discretization technique, the classical Bubnov-Galerkin technique, and the pexturbation method. These advantages are modeling versatility, reduction in total number of degrees of freedom, and simplicity of assessing the sensitivity of the thermal response to variations in the control (or path) parameters. Moreover, the proposed methods greatly alleviate the following major drawbacks of the aforementioned three techniques:

a. Excessive amounts of computer time required for the nonlinear finite-element analysis of large, complex systems 
b. Difficulty of selecting global approximation functions for the classical Bubnov-Galerkin technique

c. Small radius of convergence of the Taylor series expansions used in the classical perturbation techniques

2. The use of path derivatives as basis vectors in nonlinear steady-state thermal problems leads to accurate solutions with a small number of basis vectors. Therefore, the time required to solve the reduced equations is relatively small, and the total analysis time to a first approximation equals the time required to evaluate the basis vectors and generate the reduced equations.

3. The range of applicability of the reduction method can be greatly extended by augmenting the path derivatives of the nodal temperatures with a constant vector representing a uniform temperature rise or drop. In the problems considered, the addition of a constant vector was crucial for obtaining accurate solutions.

4. The computational effort required for generating the basis vectors can be reduced by generating only a few path derivatives (4 or 5) and then augmenting these vectors with "reciprocal vectors," in which nonzero elements are equal to the reciprocals of the nonzero values of the path derivatives. The accuracy obtained by using this set of basis vectors is comparable to that obtained by using an equal number of path derivatives.

5. The accuracy of the multiple-parameter reduction method is comparable to that of the single-parameter method. However, the former provides more information about the sensitivity of the thermal response to variations in the material properties of the structure.

Langley Research Center

National Aeronautics and Space Administration

Hampton, VA 23665

December 6, 1982 


\section{FORMULAS FOR COEFFICIENTS IN THE FINITE-ELEMENT EQUATIONS FOR INDIVIDUAL ELEMENTS}

The finite-element equations for individual elements can be written in the following form:

$$
\left[K_{I J}^{(0)}+q_{I} K_{I J K}^{(I)} T_{K}+q_{2} K_{I J K L}^{(2)} T_{K} T_{I}+q_{3} K_{I J K L N}^{(r)} T_{K} T_{L} T_{N}+K_{I J}^{(h)}\right] T_{J}=q\left(Q_{I}^{(0)}+Q_{I}^{(r)}+Q_{I}^{(h)}\right)
$$

where $\mathrm{K}_{\mathrm{IJ}}^{(0)}, \mathrm{K}_{\mathrm{IJK}}^{(1)}$, and $\mathrm{K}_{\mathrm{IJKL}}^{(2)}$ are full-system linear and normalized nonlinear conduction arrays; $K_{I J K L N}^{(r)}$ and $K_{I J}^{(h)}$ are full-system radiation and convection arrays; $Q_{I}^{(0)}$ are normalized applied heating thermal-load coefficients for the full system; and $Q_{I}^{(r)}$ and $Q_{I}^{(h)}$ are normalized radiation and convection thermal-load coefficients for the full system. The range of the uppercase subscripts (Latin index) is $l$ to $\mathrm{m}$, where $\mathrm{m}$ is the number of nodes in the element, and a repeated uppercase subscript denotes summation over the full range.

The coefficients in equation ( $A 1$ ) are defined as follows:

$$
\begin{aligned}
& \mathrm{K}_{\text {IJ }}^{(0)}=\int_{\Omega(e)} k_{\alpha \beta}^{\circ} \partial_{\alpha} N_{I} \partial_{\beta} N_{J} \mathrm{~d} \Omega \\
& \mathrm{K}_{\mathrm{IJK}}^{(1)}=\int_{\Omega} \gamma_{I} k_{\alpha \beta}^{0} \partial_{\alpha} N_{I} \partial_{\beta} N_{J} N_{K} d \Omega \\
& K_{\text {IJKL }}^{(2)}=\int_{\Omega(e)} \gamma_{2} k_{\alpha \beta}^{\circ} \partial_{\alpha} N_{I} \partial_{\beta} N_{J} N_{K} N_{L} d \Omega \\
& K_{I J K L N}^{(r)}=\int_{c_{r}^{(e)}} \sigma \varepsilon N_{I} N_{J} N_{K} N_{L} N_{N} d c
\end{aligned}
$$

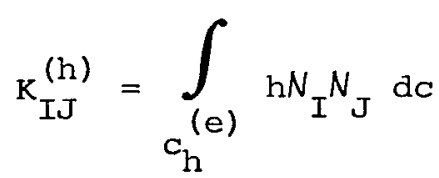




$$
\begin{aligned}
& Q_{I}^{(0)}=\int_{\Omega(e)} Q N_{I} d \Omega \\
& Q_{I}^{(r)}=\int_{c_{r}^{(e)}} a H N_{I} d c \\
& Q_{I}^{(h)}=\int_{(e)}{ }^{h T_{h} N_{I}} d c
\end{aligned}
$$

In the above equations, $N_{I}$ are shape (interpolation) functions, $\partial_{\alpha} \equiv \partial / \partial x_{\alpha}$, $\partial_{\beta} \equiv \partial / \partial x_{\beta}, \Omega^{(e)}$ is the element domain, $c_{r}^{(e)}$ is the portion of the element domain where radiant heating (or cooling) is prescribed, $c_{h}^{(e)}$ is the portion of the element domain where convective heating (or cooling) is prescribed, $Q$ is the source heat rate, $\sigma$ is the stefan-Boltzmann constant, $\varepsilon$ is the emissivity, a is the absorptivity, $H$ is the incident radiant heat $f l u x, h$ is the convection coefficient, and $\mathrm{T}_{\mathrm{h}}$ is the known convective-exchange temperature. The range of the indices $\alpha, \beta$ is 1,2 , and a repeated index denotes summation over the full range. Note that the $k$ arrays possess the following indicial symmetries: $K_{I J}^{(0)}$ and $K_{I J}^{(h)}$ are symmetric with respect to interchange of $I$ and $J ; K_{I J K}^{(1)}$ is symmetric with respect to interchange of $I$ and $J ; K_{I J K J}^{(2)}$ is symmetric with respect to interchange of $I$ and $J$ or $\mathrm{K}$ and $\mathrm{L}$; and $\mathrm{K}_{\mathrm{IJKLN}}^{(r)}$ is completely symmetric with respect to interchange of any two indices.

Elements with identical geometry and identical thermal properties have identical $\mathrm{K}$ arrays. Therefore, the $\mathrm{K}$ arrays are generated only for different elements (i.e., for elements with different geometry and/or different thermal properties). 
The reduced system of equations (eq. (11)) can be written in index notation as follows:

$$
\left[\tilde{K}_{i j}^{(0)}+q_{1} \tilde{K}_{i j k}^{(l)} \psi_{k}+q_{2} \tilde{K}_{i j k \ell}^{(2)} \psi_{k} \psi_{\ell}+q_{3} \tilde{K}_{i j k \ell n}^{(r)} \psi_{k} \psi_{\ell} \psi_{n}+\tilde{K}_{i j}^{(h)} \psi_{j}=q\left(\tilde{Q}_{i}^{(o)}+\tilde{Q}_{i}^{(r)}+\tilde{Q}_{i}^{(h)}\right)\right.
$$

The structure of these equations is similar to that of the finite-element equations for individual elements given in appendix $A$. The arrays in equation (Bl) can be expressed in terms of the full-system arrays defined in appendix $A$ and the matrix of basis vectors $\Gamma_{I i}$ as follows:

$$
\begin{aligned}
& \tilde{\mathrm{K}}_{\mathrm{ij}}^{(0)}=\sum_{\text {Elements }} \mathrm{K}_{\mathrm{IJ}}^{(0)} \Gamma_{I i} \Gamma_{J j} \\
& \tilde{K}_{i j k}^{(1)}=\sum_{\text {Elements }} \mathrm{K}_{I J K}^{(1)} \Gamma_{I i} \Gamma_{J j} \Gamma_{K k} \\
& \tilde{\mathrm{K}}_{i j k \ell}^{(2)}=\sum_{\text {Elements }} \mathrm{K}_{\mathrm{IJKL}}^{(2)} \Gamma_{I i} \Gamma_{J j} \Gamma_{K k} \Gamma_{L \ell} \\
& \tilde{\mathrm{K}}_{i j k \ell \mathrm{n}}^{(r)}=\sum_{\text {Elements }} \mathrm{K}_{I J \mathrm{KLN}}^{(r)} \Gamma_{I i} \Gamma_{J j} \Gamma_{\mathrm{Kk}} \Gamma_{L \ell} \Gamma_{\mathrm{Nn}} \\
& \tilde{\mathrm{K}}_{i j}^{(h)}=\sum_{\text {Elements }} \mathrm{K}_{\mathrm{IJ}}^{(\mathrm{h})} \Gamma_{I i} \Gamma_{J j} \\
& \tilde{Q}_{i}^{(0)}=\sum_{\text {Elements }} Q_{I}^{(0)} \Gamma_{I i} \\
& \tilde{Q}_{i}^{(r)}=\sum_{\text {Elements }} Q_{I}^{(r)} \Gamma_{I i} \\
& \tilde{Q}_{i}^{(h)}=\sum_{\text {Elements }} Q_{I}^{(h)} \Gamma_{I i}
\end{aligned}
$$


where the range of uppercase subscripts (Latin index) is $I$ to $m$ (number of nodes in the element), the range of lowercase subscripts (Latin index) is 1 to $r$ (number of reduced-system degrees of freedom), and a repeated index in the same term denotes summation over the full range.

Note that the indicial symmetries exhibited by the full-system arrays are retained in the reduced system as follows: $\tilde{\mathrm{K}}_{i j}^{(0)}$ and $\tilde{\mathrm{K}}_{i j}^{(\mathrm{h})}$ are symmetric arrays; $\tilde{K}_{i j k}^{(1)}$ is symmetric with respect to interchange of $i$ and $j ; \tilde{K}_{i j k l}^{(2)}$ is symmetric with respect to interchange of $i$ and $j$ or $k$ and $l$; and $\tilde{K}_{i j k l n}^{(r)}$ is completely symmetric with respect to the interchange of any two indices. 
The path derivatives in equations (16) and (17) are obtained by successive differentiation of the governing finite-element equations (eq. (1)) with respect to the path parameter $q$ in the single-parameter reduction method and with respect to the parameters $q_{1}, q_{2}$, and $q_{3}$ in the three-parameter reduction method. The explicit forms of the recursion relations for individual elements are given in this appendix.

\section{Single-Parameter Reduction Method}

The recursion relations for the path derivatives can be written in the following compact form:

$$
\left.\left[K_{I J}^{(0)}+K_{I J}^{(h)}\right] \frac{\partial^{a} T_{J}}{\partial q^{a}}\right|_{q=0}=R_{I}^{(a)}
$$

where $I$ and $J$ range from $I$ to $m$ (number of nodes in the element), a ranges from 1 to $r$ (number of reduced-system degrees of freedom), and the explicit forms of the components of the right-hand side $R_{I}^{(a)}$ are given in table $\mathrm{Cl}$.

TABLE C1.- EXPLICIT FORMS OF COMPONENTS OF $\mathrm{R}_{I}^{(a)}$ FOR SINGLE-PARAMETER METHOD

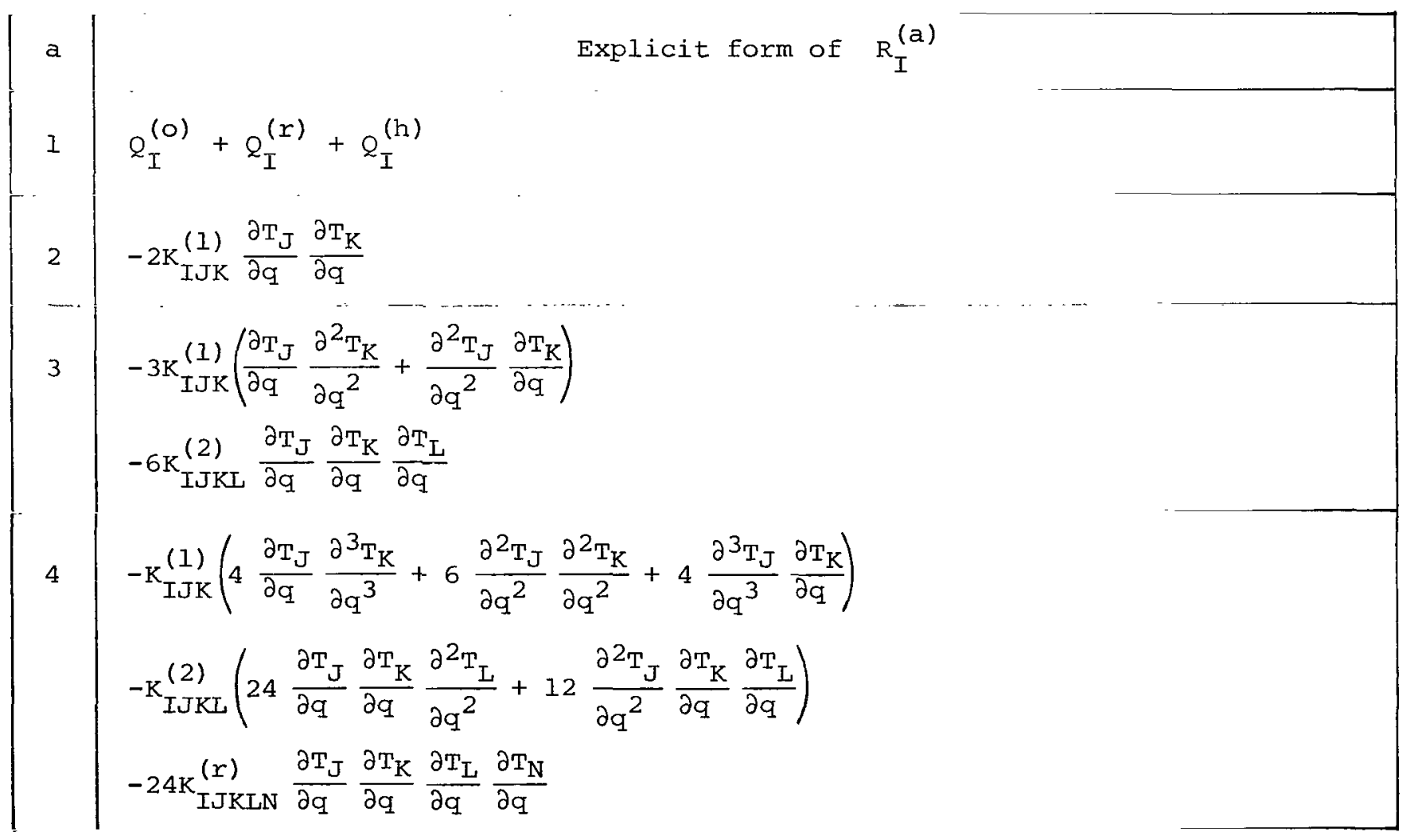


APPENDIX C

\section{TABLE Cl.- Continued}

\begin{tabular}{|c|c|}
\hline$a$ & Explicit form of $R_{I}^{(a)}$ \\
\hline 5 & 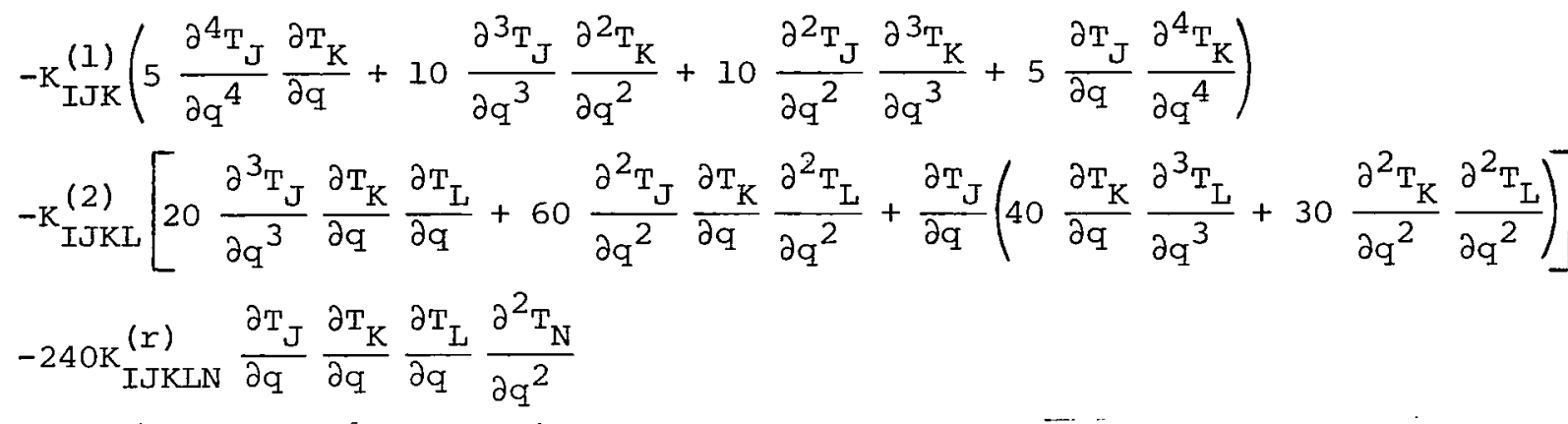 \\
\hline 6 & 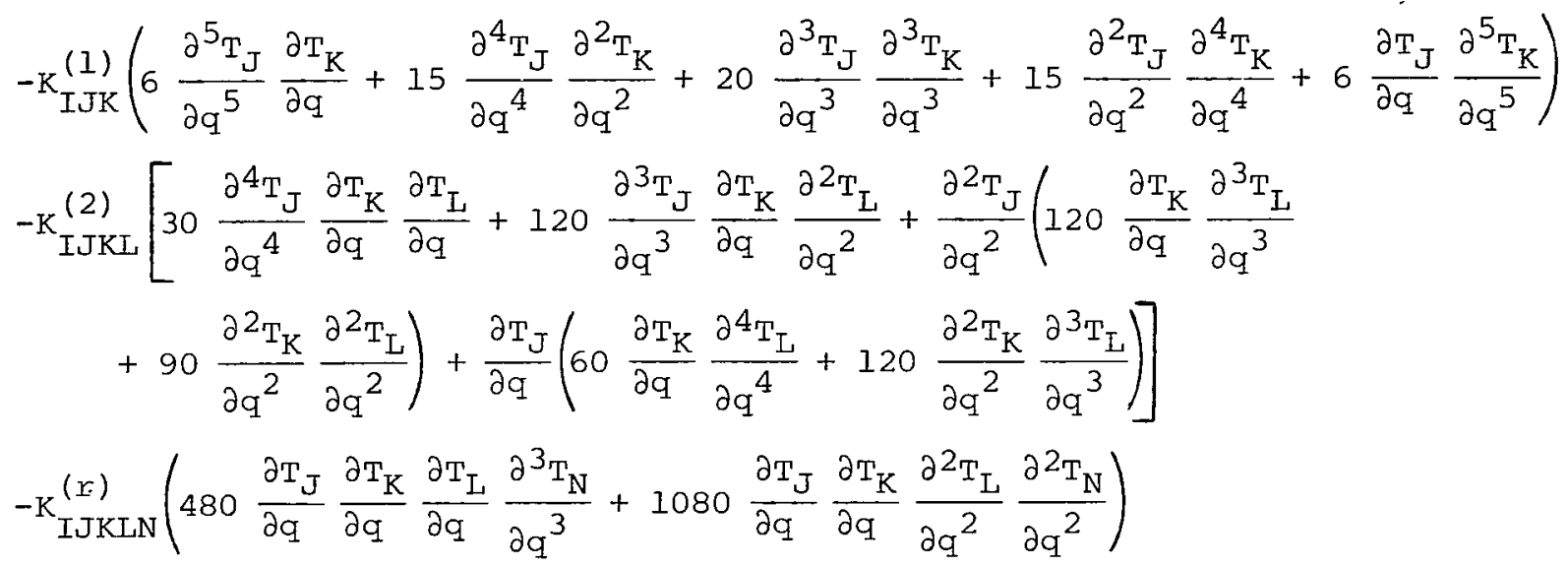 \\
\hline 7 & 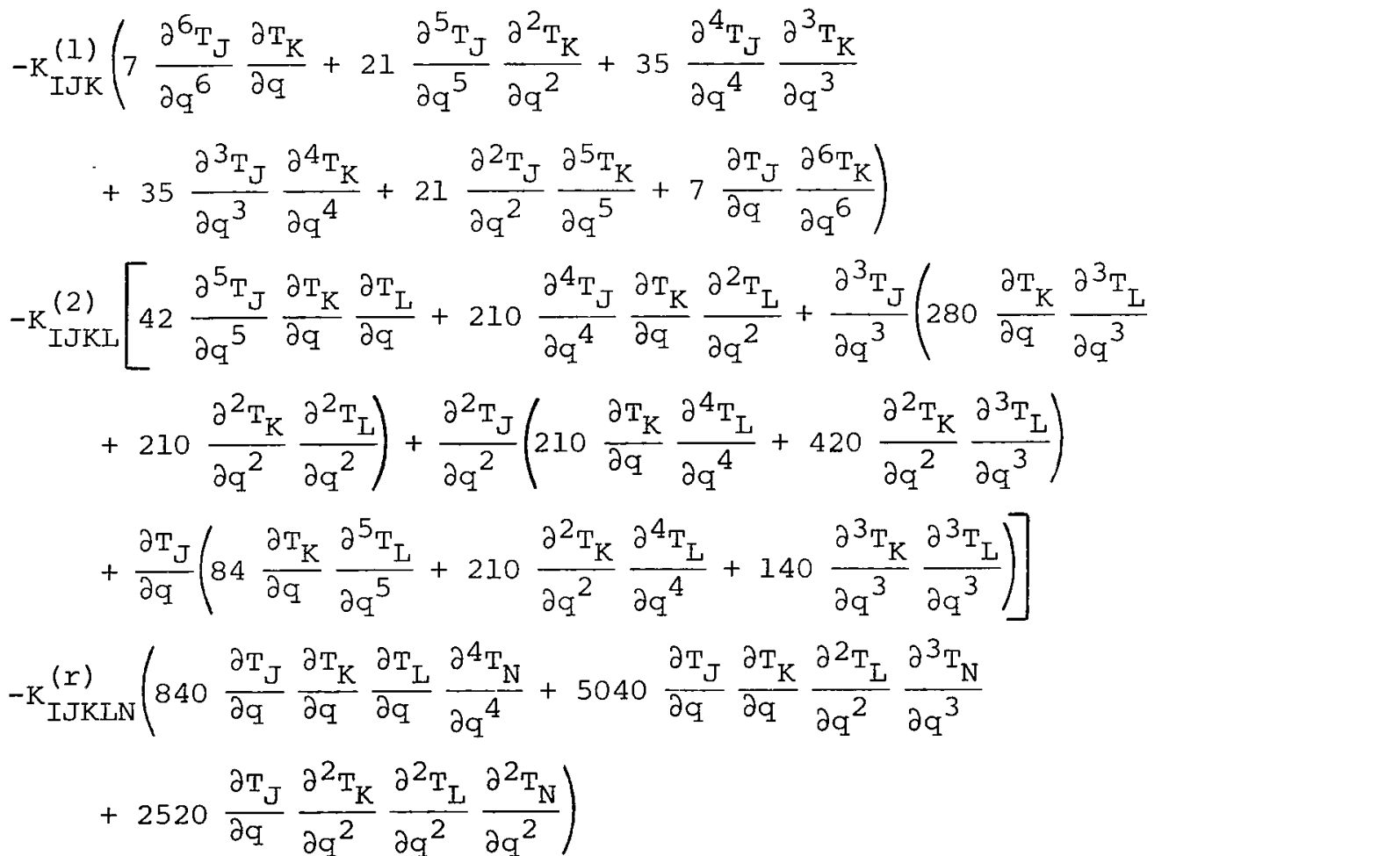 \\
\hline
\end{tabular}


TABLE Cl.- Concluded

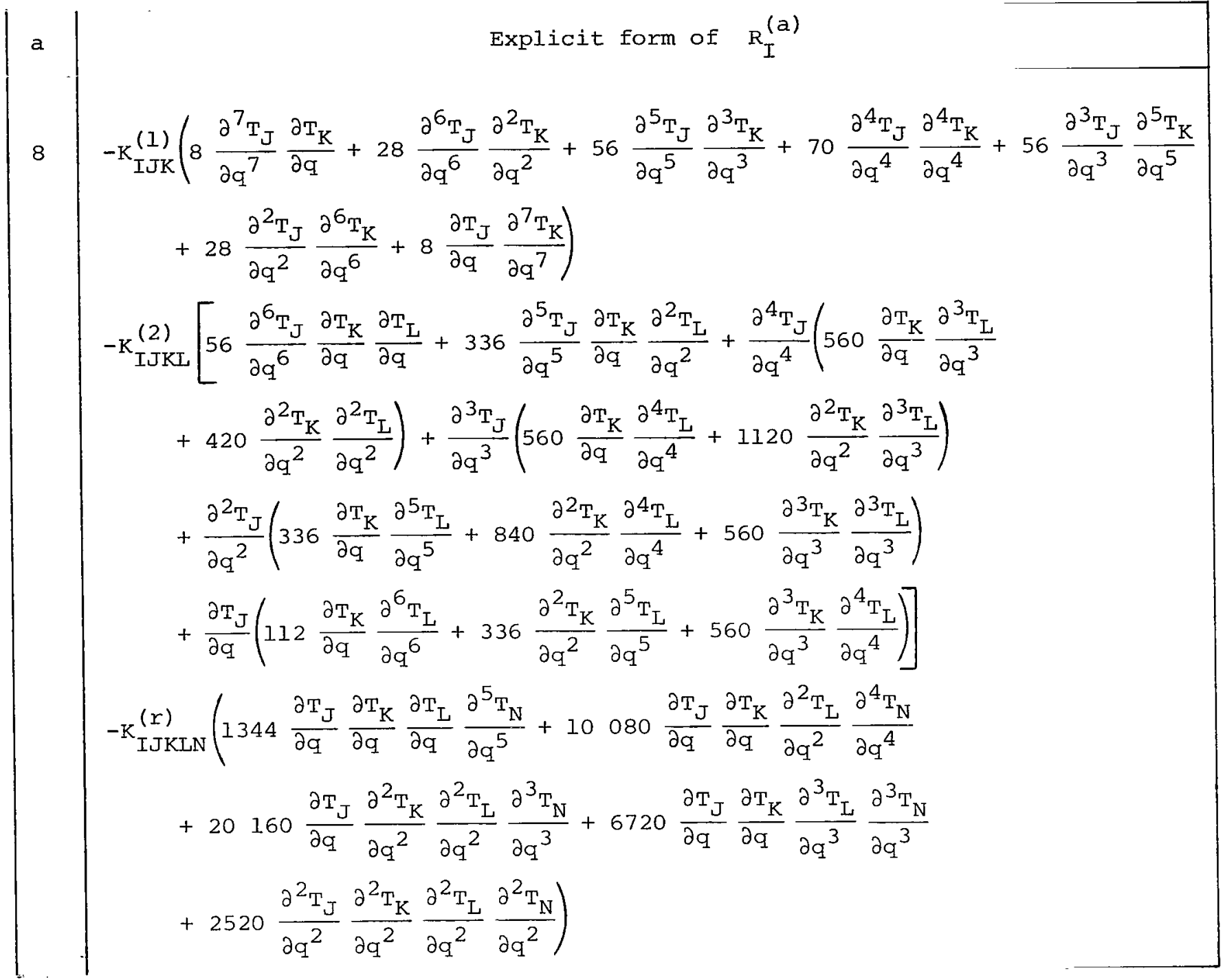

Three-Parameter Reduction Method

The recursion relations for the individual elements have the following form:

$$
\left.\left[K_{I J}^{(0)}+K_{I J}^{(h)}\right] \frac{\partial^{p+s+t_{T}}}{\partial q_{1}^{p} \partial q_{2}^{s} \partial q_{3}^{t}}\right|_{q_{1}=q_{2}=q_{3}=0}=R_{I}^{(a)}
$$

where $I$ and $J$ range from 1 to $m$. The total number of $p+s+t$ combinations is $r$, and the explicit forms of the components of the right-hand side $R_{I}^{(a)}$ are given in table $\mathrm{C} 2$. 
TABLE C2.- EXPLICIT FORMS OF COMPONENTS OF $R_{I}^{(a)}$ FOR THREE-PARAMETER METHOD

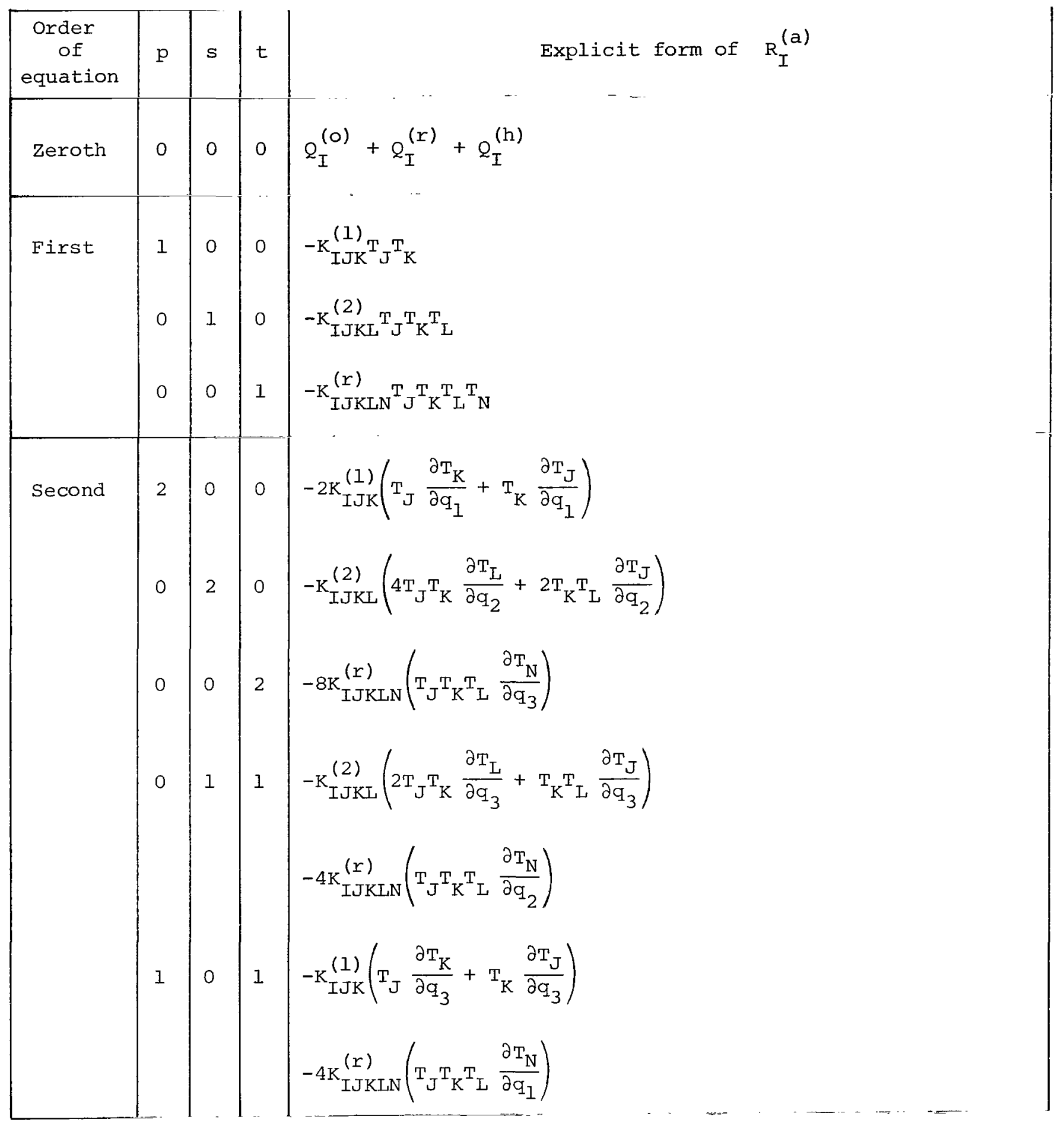


APPENDIX C

TABLE C2.- Continued

\begin{tabular}{|c|c|c|c|c|}
\hline $\begin{array}{l}\text { Order } \\
\text { of } \\
\text { equation }\end{array}$ & $\mathrm{p}$ & $s$ & $t$ & Explicit form of $R_{I}^{(a)}$ \\
\hline Second & 2 & 0 & 1 & 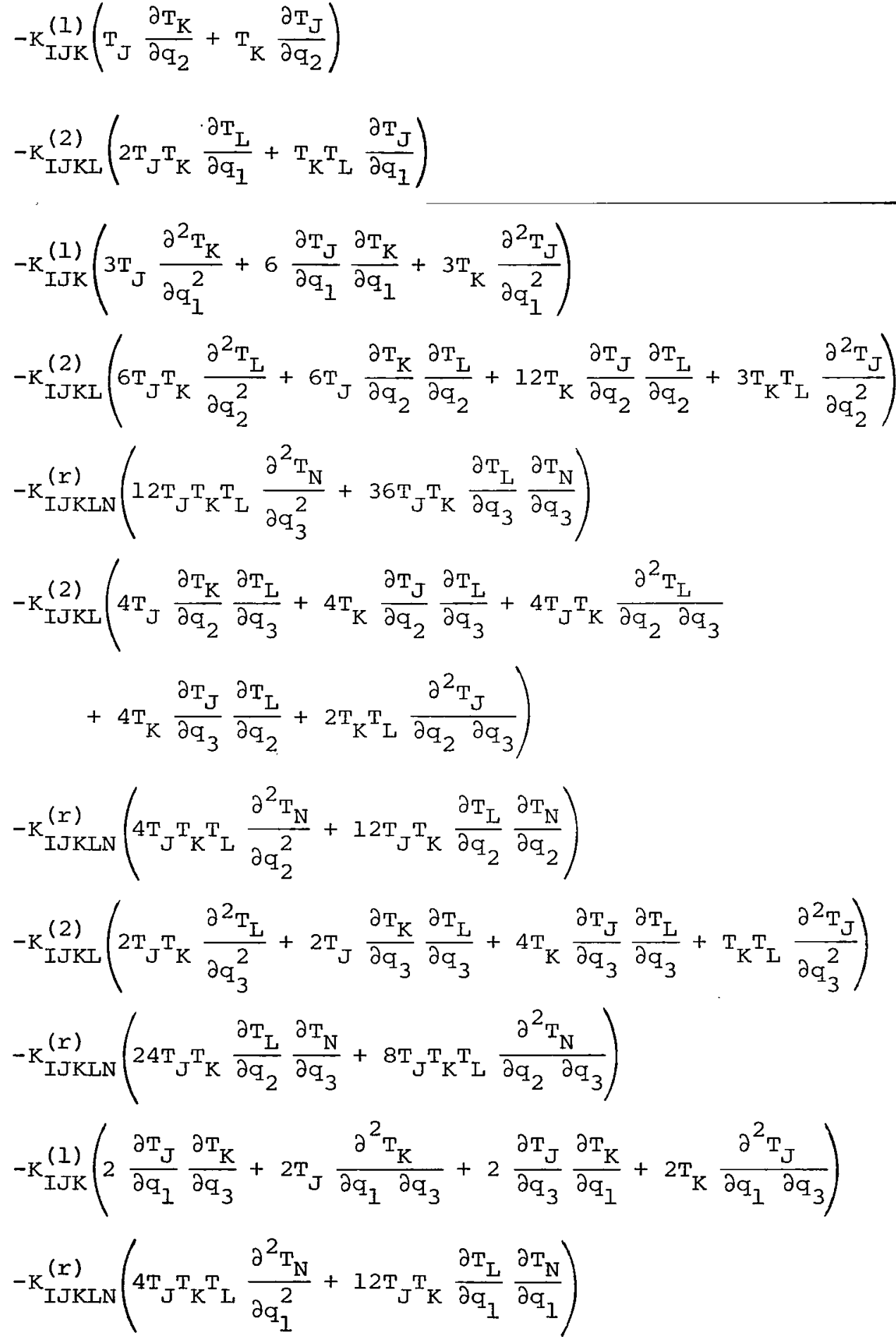 \\
\hline
\end{tabular}


APPENDIX C

TABLE C2.- Concluded

\begin{tabular}{|c|c|c|c|c|}
\hline $\begin{array}{l}\text { Order } \\
\text { of } \\
\text { equation }\end{array}$ & $p$ & $\mathrm{~s}$ & $t$ & Explicit form of $R_{I}^{(a)}$ \\
\hline Third & 1 & 1 & 0 & 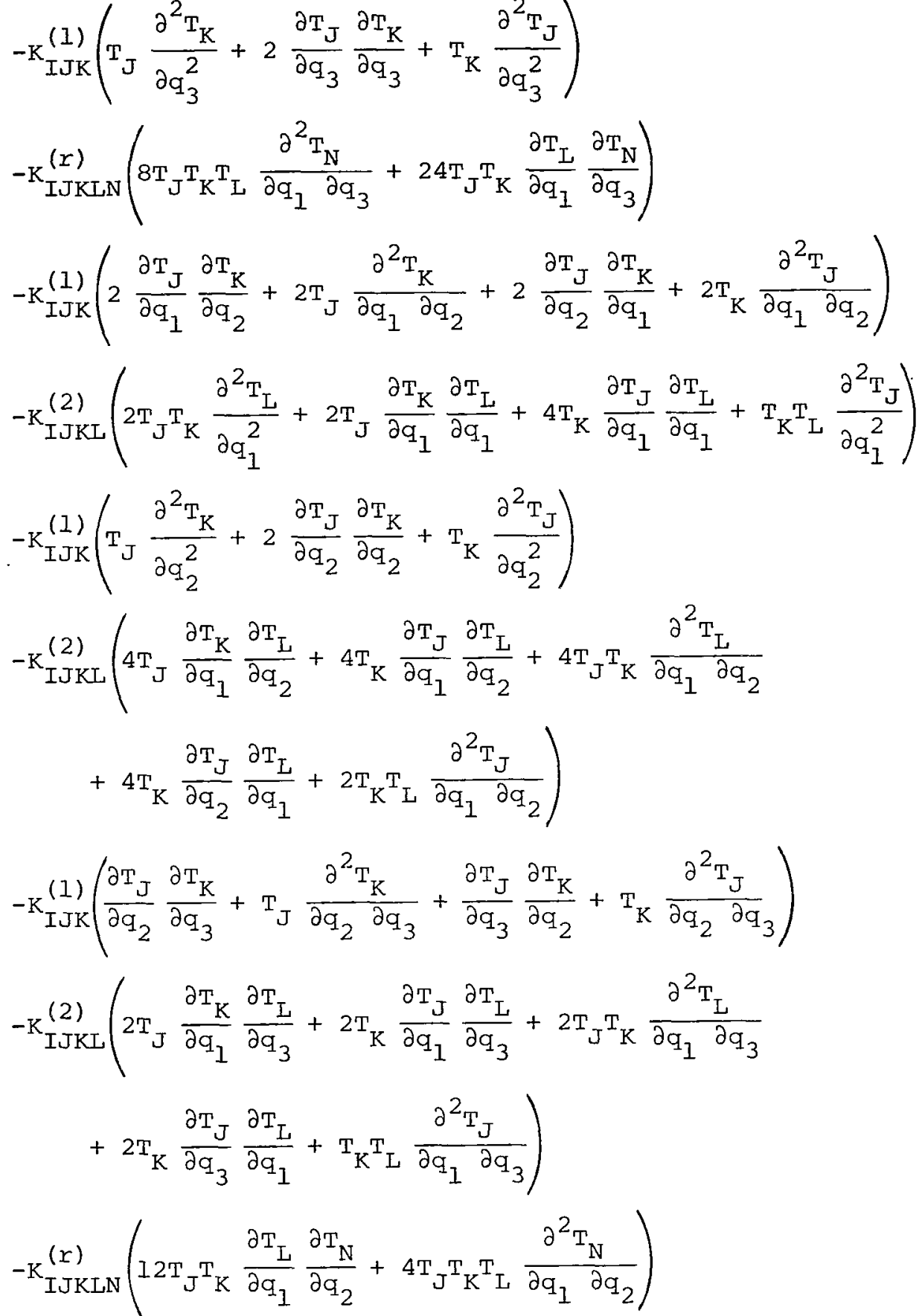 \\
\hline
\end{tabular}




\section{APPENDIX C}

In tables $\mathrm{Cl}$ and $\mathrm{C} 2$ a repeated uppercase Latin index denotes summation over the range 1 to $\mathrm{m}$. The evaluation of the right-hand-side vectors listed in tables $\mathrm{cl}$ and $\mathrm{C} 2$ involves contraction of $K$ arrays with basis vectors. Many of the contracted arrays reappear in subsequent right-hand sides. For computational efficiency, the contracted arrays which are common to more than one right-hand side are generated once and stored for subsequent use. Note that the coefficient matrix on the lefthand sides of equations (Cl) and (C2), which must be factored, is the same for each of the path derivatives. Hence, after assembly, the global matrix is factored only once regardless of the number of path derivatives generated. 


\section{APPENDIX D}

TREATMENT OF PRESCRIBED BOUNDARY TEMPERATURES

In the case of prescribed nonzero values for the boundary temperatures, it is convenient to partition the vector of nodal temperatures $\{\mathrm{T}\}$ as follows:

$$
\{\mathrm{T}\}=\left\{\begin{array}{c}
\mathrm{T}_{\mathrm{f}} \\
\mathrm{T}_{\mathrm{p}}
\end{array}\right\}
$$

where $\left\{\mathrm{T}_{f}\right\}$ and $\left\{\mathrm{T}_{\mathrm{p}}\right\}$ are the vectors of free and prescribed nonzero nodal temperatures. The prescribed zero nodal temperatures and their associated equations are eliminated from equation ( 1 ). The prescribed nonzero nodal temperatures are assumed to be proportional to the parameter $q$, that is,

$$
\left\{T_{p}\right\}=q\{z\}
$$

\section{Governing Finite-Element Equations} as

Equation (I) can be conveniently partitioned into two sets of matrix equations

$$
\left\{\begin{array}{l}
f_{f}\left(T_{f}, T_{p}\right) \\
f_{p}\left(T_{f}, T_{p}\right)
\end{array}\right\}=\left[\begin{array}{ll}
K_{f f}(T) & K_{f p}(T) \\
K_{p f}(T) & K_{p p}(T)
\end{array}\right]\left\{\begin{array}{l}
T_{f} \\
T_{p}
\end{array}\right\}-q\left\{\begin{array}{l}
Q_{f} \\
Q_{p}
\end{array}\right\}=0
$$

In the absence of heat sources and radiation and convection thermal loads, $\left\{g_{\mathrm{f}}\right\}=0$ and $\left\{\mathrm{g}_{\mathrm{p}}\right\}$ equals the equivalent thermal loads associated with the prescribed temperatures $\left\{\mathrm{T}_{\mathrm{p}}\right\}$. The first set of equations in equation (D3) is used to determine $\left\{\mathrm{T}_{f}\right\}$ and the second set can then be used to evaluate the vector $\left\{g_{p}\right\}$.

Basis Vectors and the Reduced System of Equations

The matrix $[\Gamma]$ in equation (10) can be conveniently partitioned as follows:

$$
[\Gamma]=\left[\begin{array}{l}
\Gamma_{\mathrm{f}} \\
\Gamma_{\mathrm{p}}
\end{array}\right]
$$


where

$$
\left[\begin{array}{c}
\Gamma_{f} \\
\Gamma_{p}
\end{array}\right]=\left[\left\{\begin{array}{c}
0 \\
z
\end{array}\right\},\left\{\begin{array}{c}
\frac{\partial \mathrm{T}_{f}}{\partial \mathrm{q}} \\
0
\end{array}\right\},\left\{\begin{array}{c}
\frac{\partial^{2} \mathrm{~T}_{f}}{\partial \mathrm{q}^{2}} \\
0
\end{array}\right\}, \begin{array}{c}
\cdots \\
\ldots
\end{array}\right]
$$

for the single-parameter case, and

$$
\left[\begin{array}{c}
\Gamma_{f} \\
\Gamma_{P}
\end{array}\right]=\left[\left\{\begin{array}{l}
0 \\
z
\end{array}\right\},\left\{\begin{array}{c}
\frac{\partial \mathrm{T}_{f}}{\partial q_{1}} \\
0
\end{array}\right\},\left\{\begin{array}{c}
\frac{\partial \mathrm{T}_{f}}{\partial \mathrm{q}_{2}} \\
0
\end{array}\right\},\left\{\begin{array}{c}
\frac{\partial \mathrm{T}_{f}}{\partial \mathrm{q}_{3}} \\
0 \\
0 \\
\ldots
\end{array}\right]\right.
$$

for the three-parameter case.

The corresponding reduced-system equations are given by equation (11) with $\{\tilde{Q}\}$ given by

$$
\{\tilde{Q}\}=[\Gamma]^{T}\left\{\begin{array}{l}
Q_{F} \\
Q_{p}
\end{array}\right\}
$$

Note that when $\left\{Q_{f}\right\}=0,\{\tilde{Q}\}$ has only one nonzero component, namely $\tilde{Q}_{1}$. Equation (11) is solved for the reduced unknowns $\{\psi\}$ subject to the condition $\psi_{1}=q$. Whenever desired, the basis vectors in equations (D5) and (D6) are augmented by the constant and reciprocal vectors defined in the paper. 
EXACT SOLUTION FOR TWO-DIMENSIONAL STEADY-STATE CONDUCTION IN A SQUARE PLATE WITH TEMPERATURE-DEPENDENT THERMAL CONDUCTIVITIES

The exact solution for the two-dimensional steady-state conduction in a square isotropic plate with temperature-dependent thermal conductivities can be obtained by using the Kirchhoff transformation (see page 11 of ref. 11), which involves the following substitution:

$$
U(T)=\frac{l}{k^{\circ}} \int_{0}^{T} k(T) d T
$$

For quadratic variation of conductivity with temperature of the form $k(T)=k^{\circ}\left(1+\gamma_{1} T+\gamma_{2} T^{2}\right)$, equation (El) becomes

$$
U(T)=T\left(I+\frac{1}{2} \gamma_{1} T+\frac{1}{3} \gamma_{2} T^{2}\right)
$$

The governing differential equation for heat conduction in the plate is linear in the transformed variable $U(T)$. The expression of $U(T)$ which satisfies the boundary conditions shown in figure 1 can be written in the following form:

$$
\mathrm{U}=\sum_{\mathrm{m}=1,3,5, \ldots}^{\infty} \mathrm{A}_{\mathrm{m}} \sin \frac{\mathrm{m} \pi \mathrm{x}_{1}}{\mathrm{~L}} \sinh \frac{\mathrm{m} \pi \mathrm{x}_{2}}{\mathrm{~L}}
$$

where $I$ is the side length of the plate and

$$
\begin{aligned}
A_{m}= & \frac{2}{L \sinh (m \pi)} \int_{0}^{L} \sin \frac{m \pi x_{1}}{L}\left[q \sin \frac{\pi x_{1}}{L}\right. \\
& \left.+\frac{1}{2} \gamma_{1}\left(q \sin \frac{\pi x_{1}}{L}\right)^{2}+\frac{1}{3} \gamma_{2}\left(q \sin \frac{\pi x_{1}}{L}\right)^{3}\right] d x_{1}
\end{aligned}
$$

Once the coefficients $A_{m}$ have been evaluated and $U$ is determined from equation (E3), the value of the temperature $T$ at each point can be obtained by solving the cubic equation (eq. (E2)).

In the present study, 10 terms were included in the series expansion of $U$ (eq. (E3)), with an estimated maximum truncation error of $1 \times 10^{-3}$ for the temperature $T$. The computerized symbolic and algebraic manipulation system MACSYMA (ref. 12) was used in the computation. 


\section{REFERENCES}

1. Lewis, R. W.; and Morgan, K., eds.: Numerical Methods in Thermal Problems. Pineridge Press, Ltd., c.1979.

2. Lewis, R. W.; Morgan, K.; and Zienkiewicz, O. C.: Numerical Methods in Heat Transfer. John Wiley \& Sons, c.1981.

3. Adelman, Howard M., compiler: Computational Aspects of Heat Transfer in structures. NASA CP-2216, 1982.

4. Noor, Ahmed K.: Survey of Computer Programs for Heat Transfer Analysis. Computational Aspects of Heat Transfer in Structures, NASA CP-2216, 1982, pp. 487-561.

5. Noor, Ahmed K.; and Peters, Jeanne M.: Reduced Basis Technique for Nonlinear Analysis of Structures. AIAA J., vol. 18, no. 4, Apr. 1980, pp. 455-462.

6. Noor, Ahmed K.: Recent Advances in Reduction Methods for Nonlinear Problems. Comput. \& Struct., vol. 13, no. 1-3, June 1981, pp. 31-44.

7. Shore, Charles P.: Application of the Reduced Basis Method to Nonlinear Transient Thermal Analysis. Research in Structural and Solid Mechanics - 1982, NASA CP-2245, 1982, pp. 49-65.

8. Aziz, A.; and Benzies, J. Y.: Application of Perturbation Techniques to HeatTransfer Problems with Variable Thermal Properties. Int. J. Heat \& Mass Transfer, vol. 19, no. 3, Mar. 1976, pp. 271-276.

9. Padovan, J.: Steady Conduction of Heat in Linear and Nonlinear Fully Anisotropic Media by Finite Elements. Trans. ASME, Ser. C: J. Heat Transfer, vol. 96, no. 3, Aug. 1974, pp. 313-318.

10. Ko, William L.; Quinn, Robert D.; Gong, Leslie; Schuster, Lawrence S.; and Gonzales, David: Reentry Heat Transfer Analysis of the Space Shuttle Orbiter. Computational Aspects of Heat Transfer in Structures, NASA CP-2216, 1982, pp. $295-325$.

11. Carslaw, H. S.; and Jaeger, J. C.: Conduction of Heat in Solids. Second ed., Oxford Univ. Press, Inc., 1959.

12. Mathlab Group: MACSYMA Reference Manual. Version Nine, Second Printing. Massachusetts Inst. Technol., Dec. 1977. 


\section{SYMBOLS}






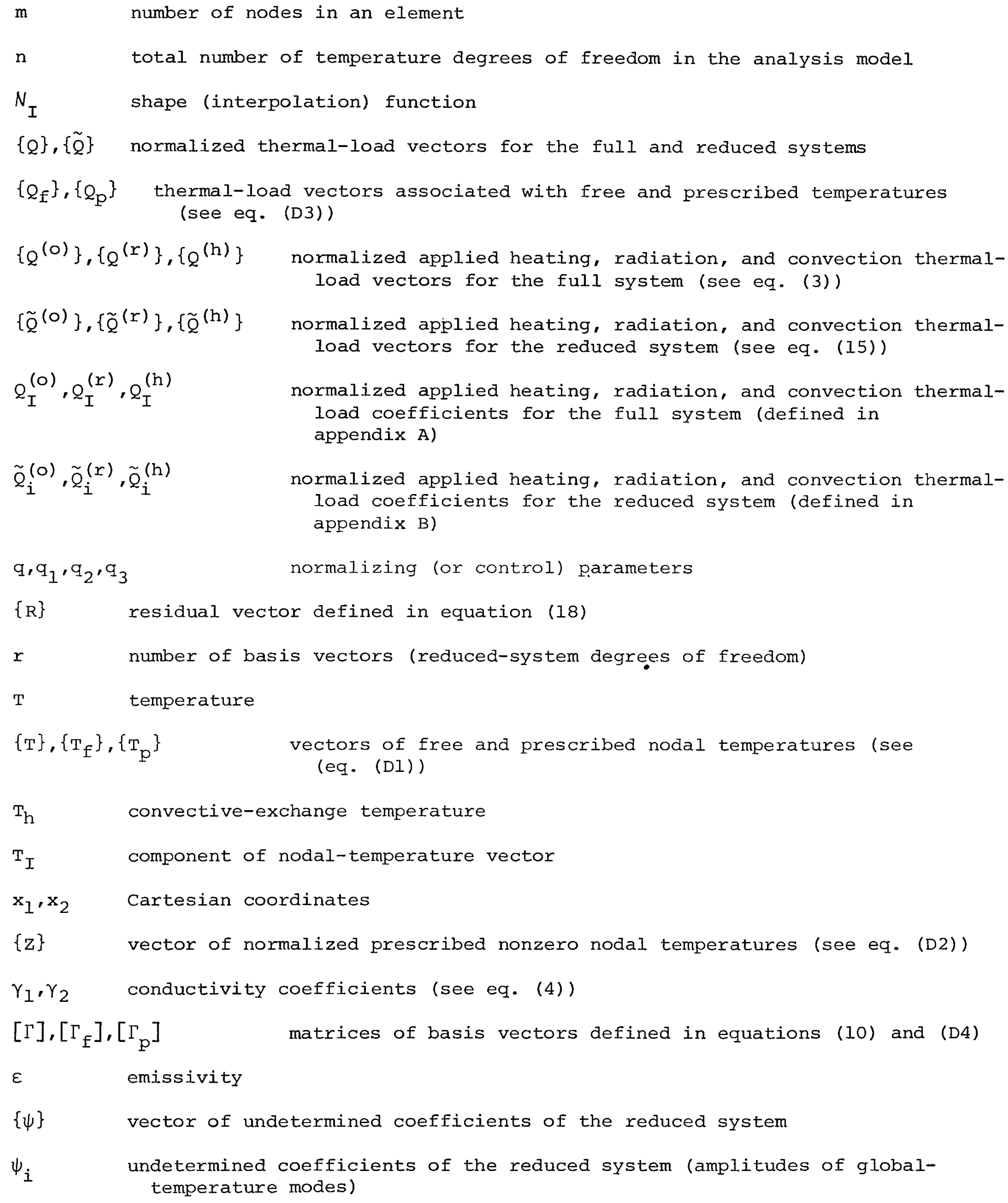




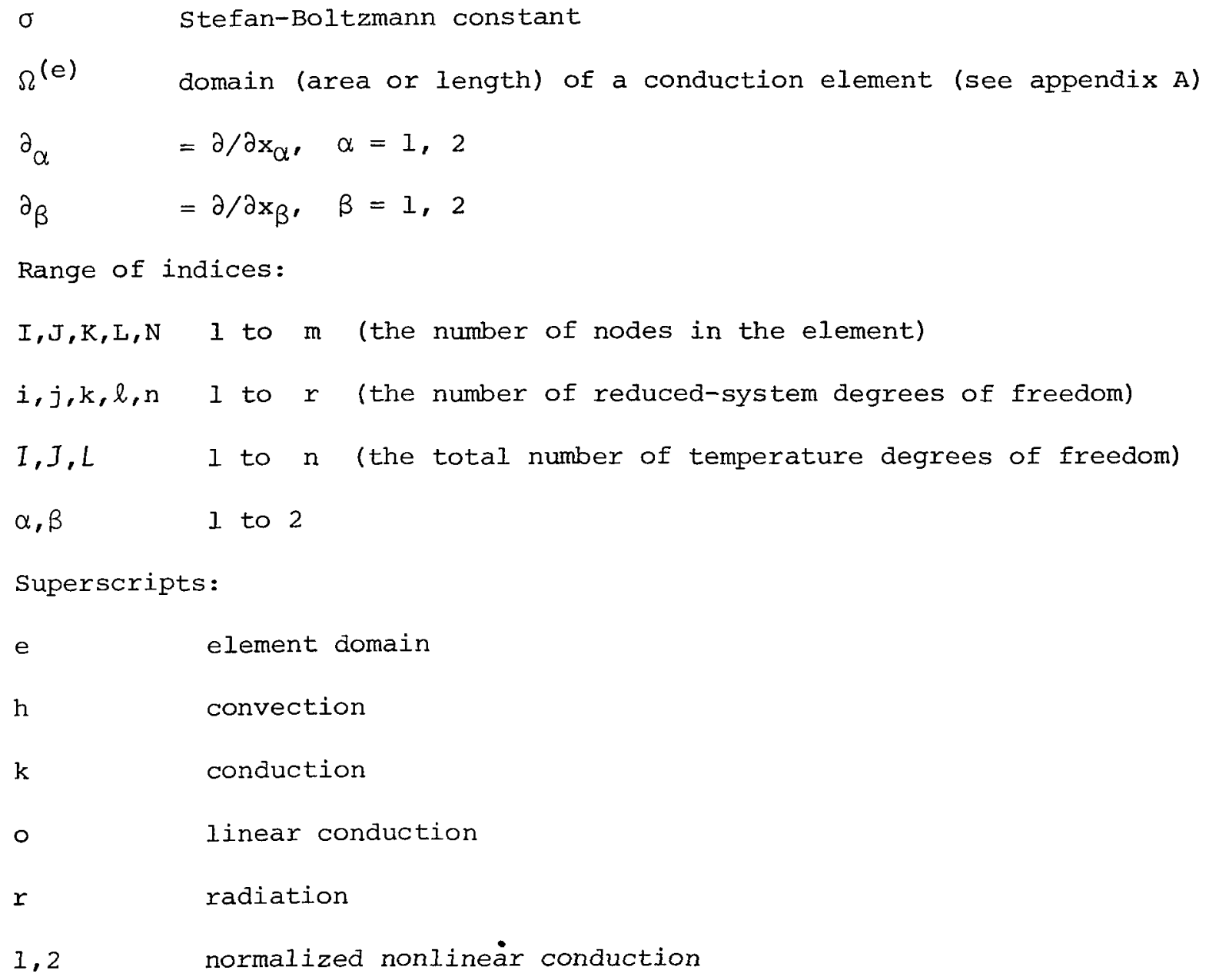




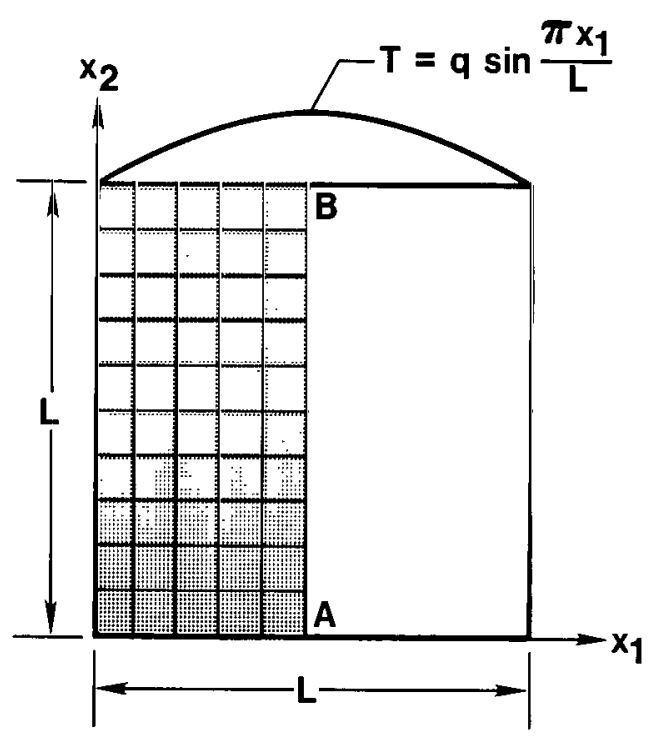

$$
\begin{aligned}
& k=k^{\circ}\left(1+\gamma_{1} \mathrm{~T}+\gamma_{2} \mathrm{~T}^{2}\right) \\
& k^{\circ}=1.0 \mathrm{~W} / \mathrm{m}-\mathrm{K} \\
& \gamma_{1}=8.333 \times 10^{-3} \\
& \gamma_{2}=1.3889 \times 10^{-4} \\
& \mathrm{~L}=1.0 \mathrm{~m}
\end{aligned}
$$
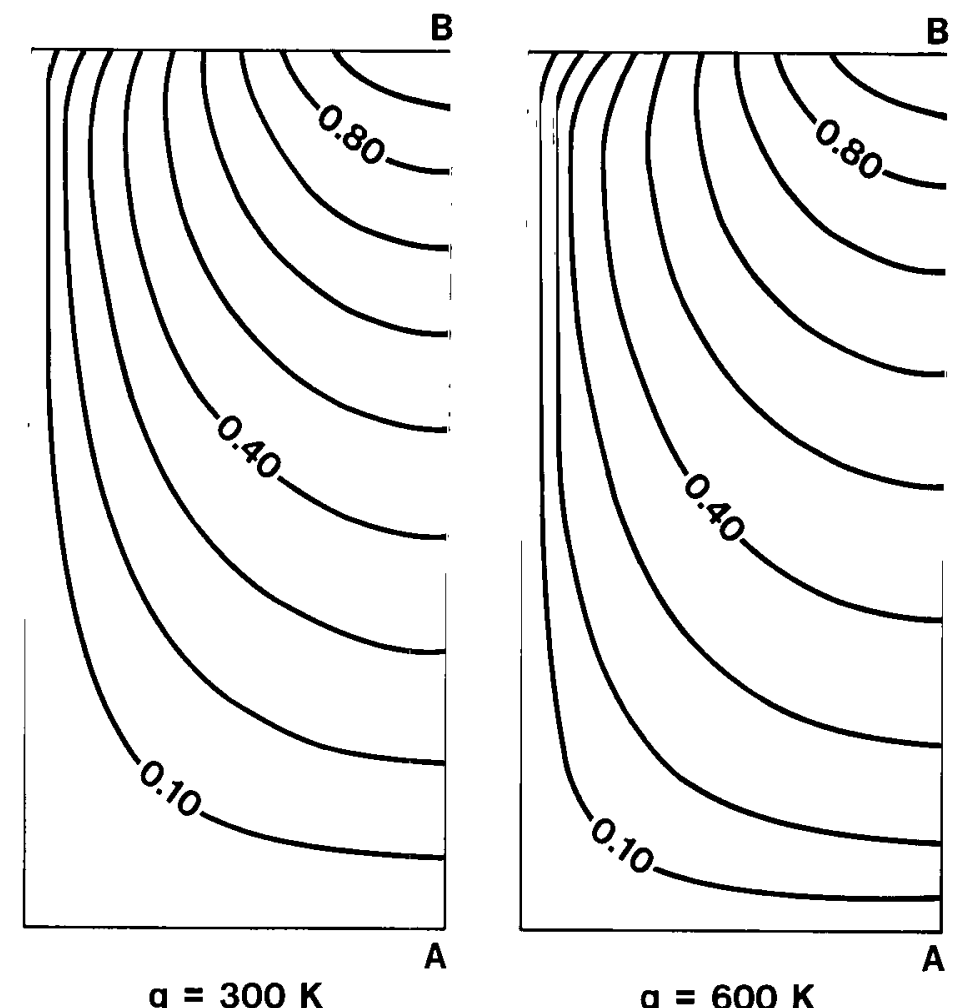

$q=600 \mathrm{~K}$

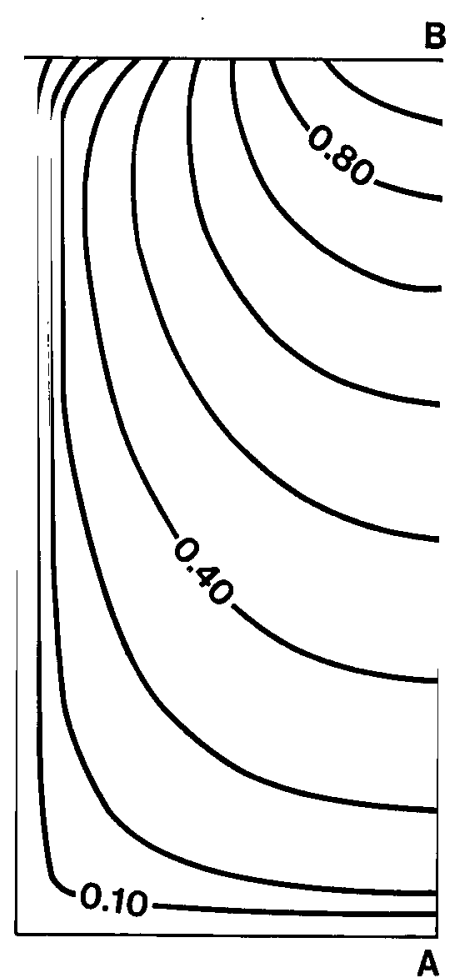

$q=2400 K$

Figure 1.- Finite-element model and normalized contour plots for temperatures at $\mathrm{q}=300 \mathrm{~K}, 600 \mathrm{~K}$, and $2400 \mathrm{~K}$ in a square plate with temperature-dependent thermal conductivities. 

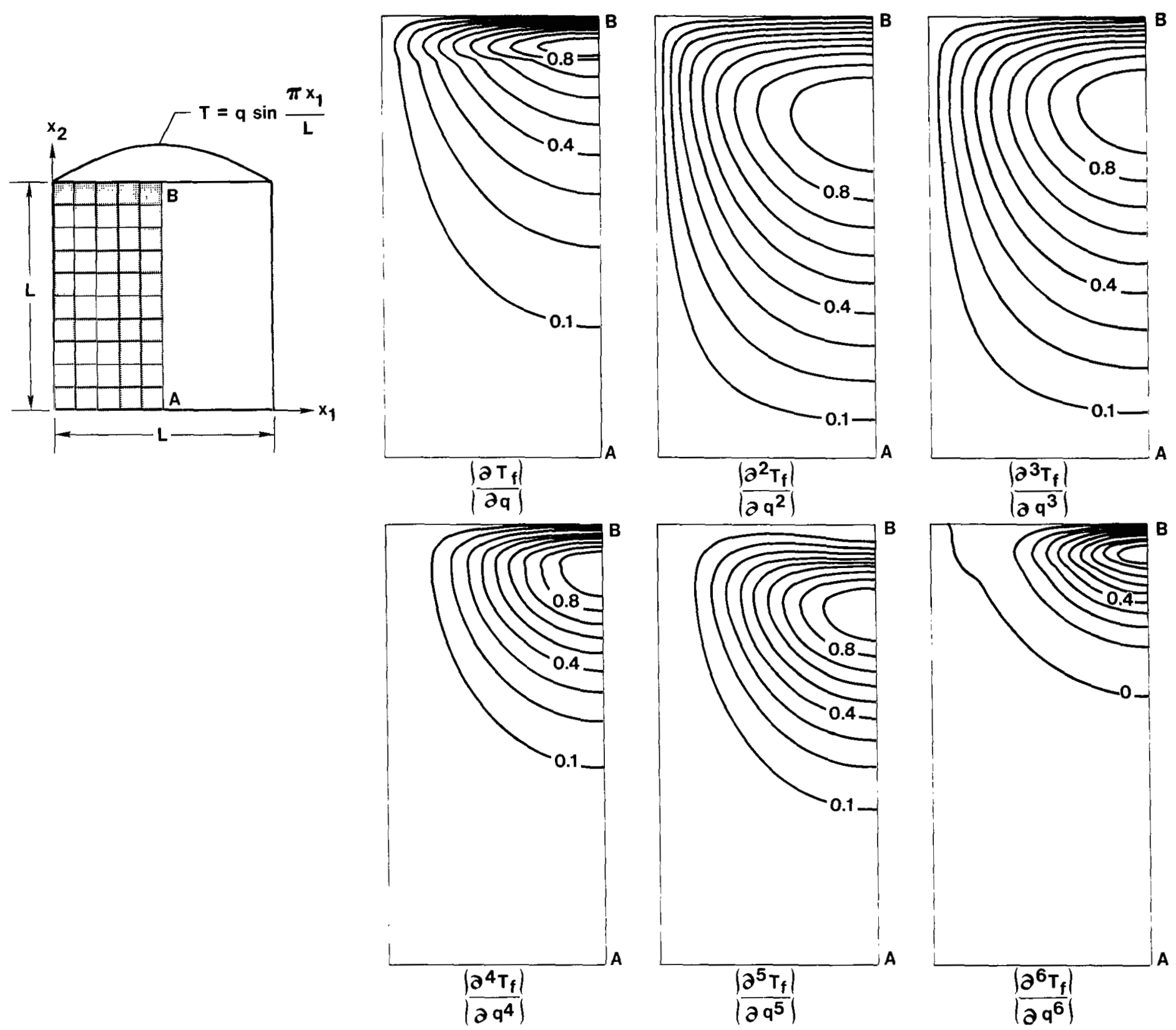

Figure 2.- Normalized contour plots for the first six path derivatives for square plate with temperature-dependent thermal conductivities. 


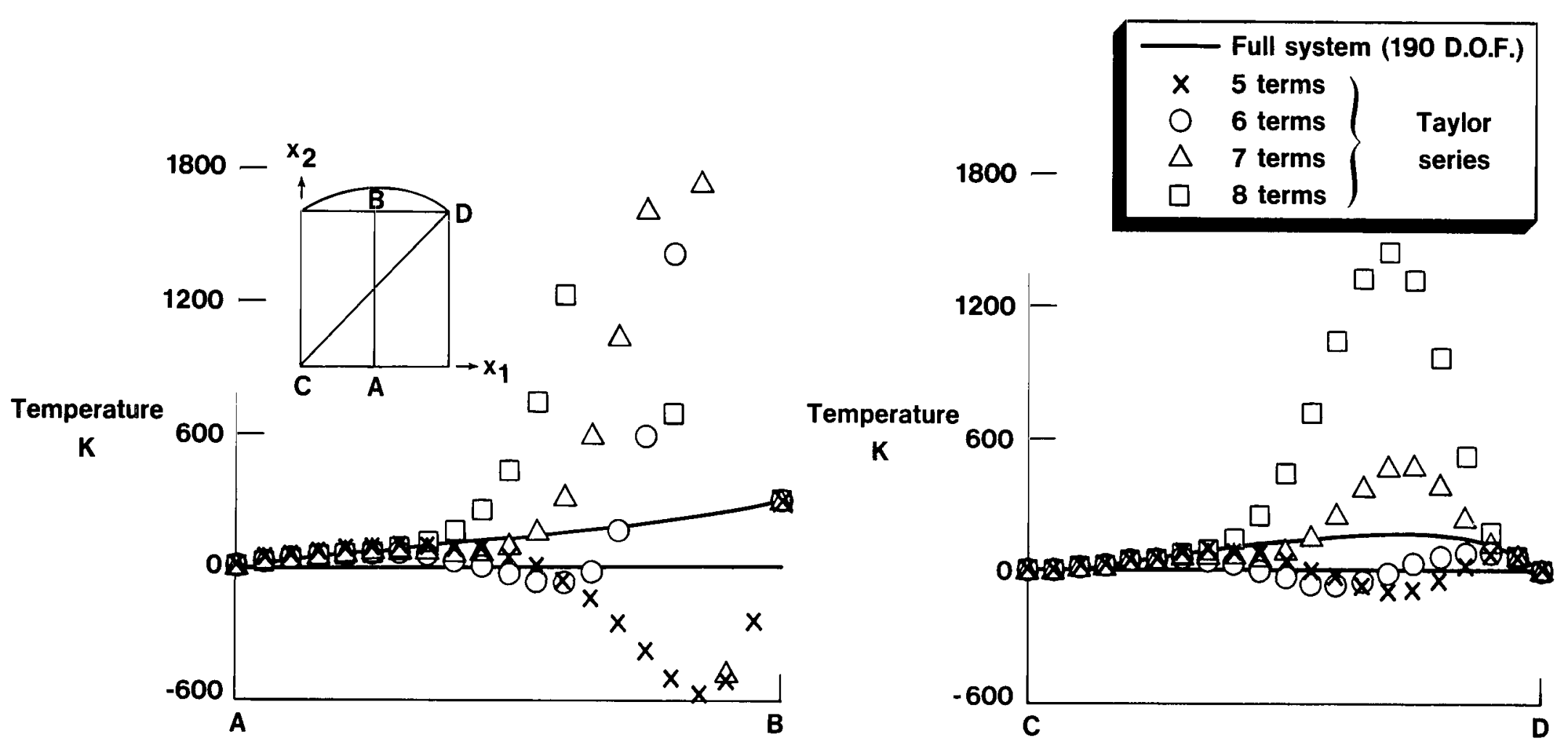

Figure 3.- Accuracy of solutions obtained by using Taylor series expansion for square plate with temperature-dependent thermal conductivities. $q=300 \mathrm{~K}$. 

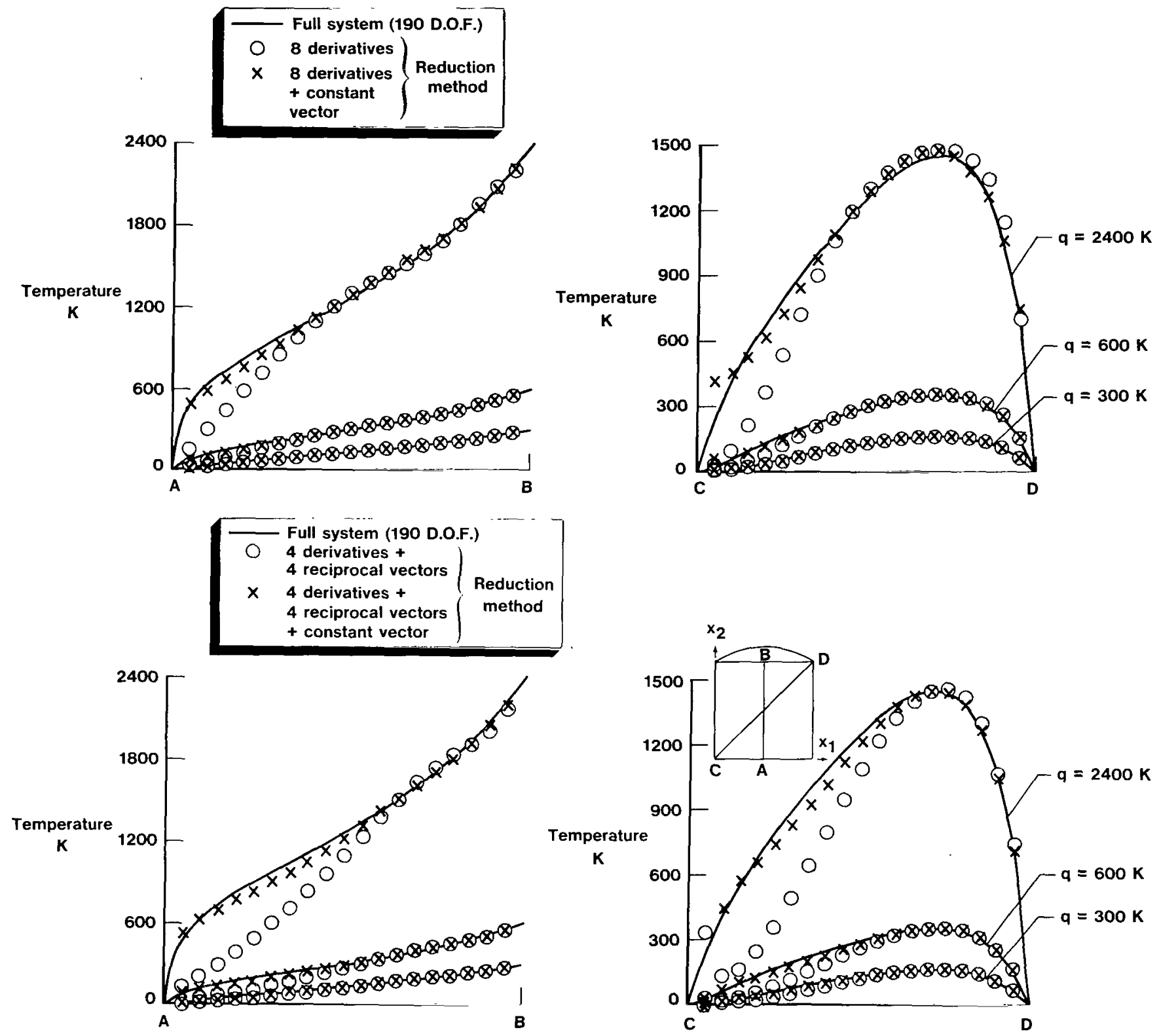

Figure 4.- Accuracy of solutions obtained by using different sets of basis vectors for square plate with temperature-dependent thermal conductivities. 


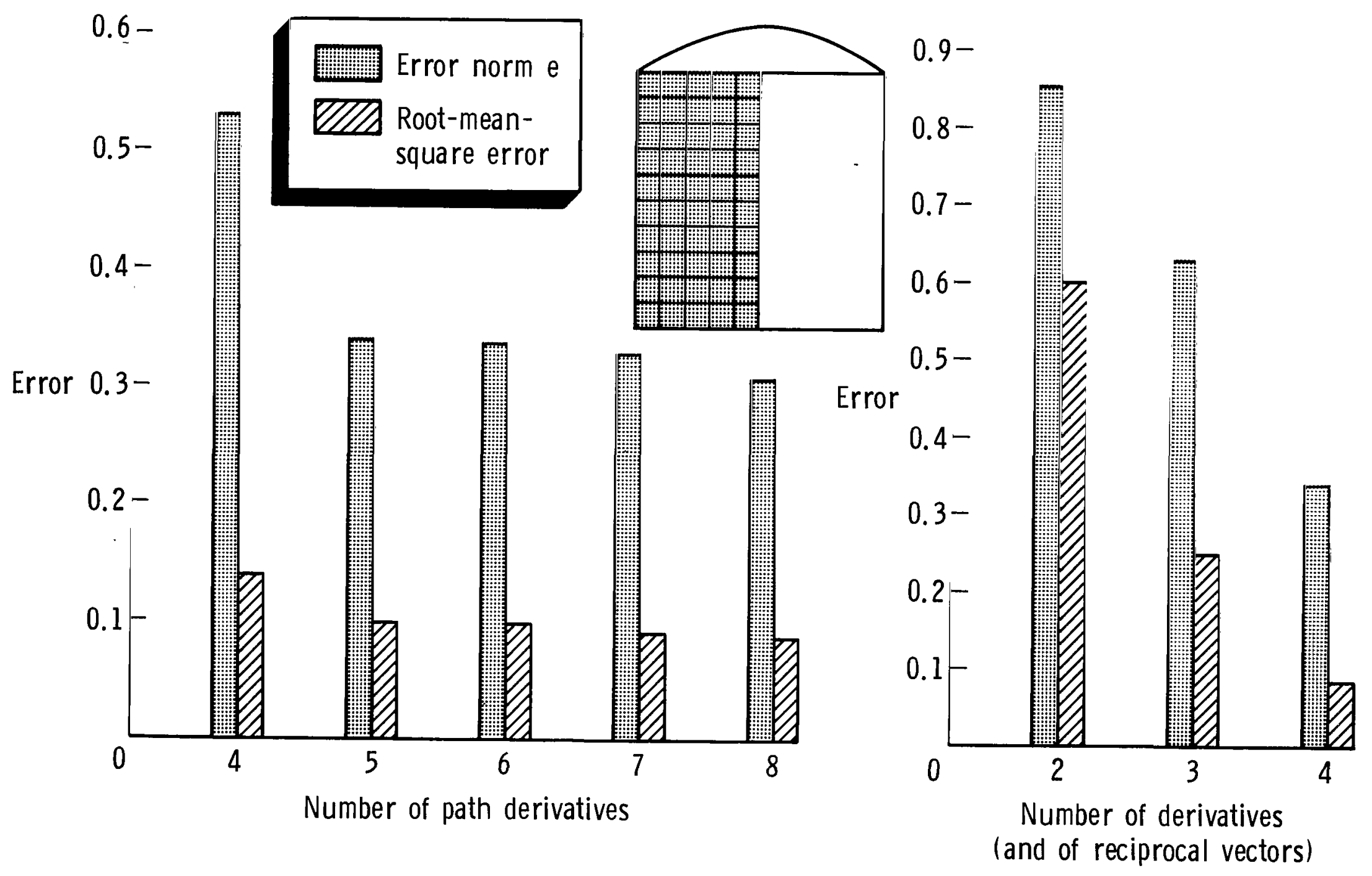

Figure 5.- Accuracy and convergence of solutions obtained with different sets of basis vectors (including the constant vector) for square plate with temperature-dependent thermal conductivities. 

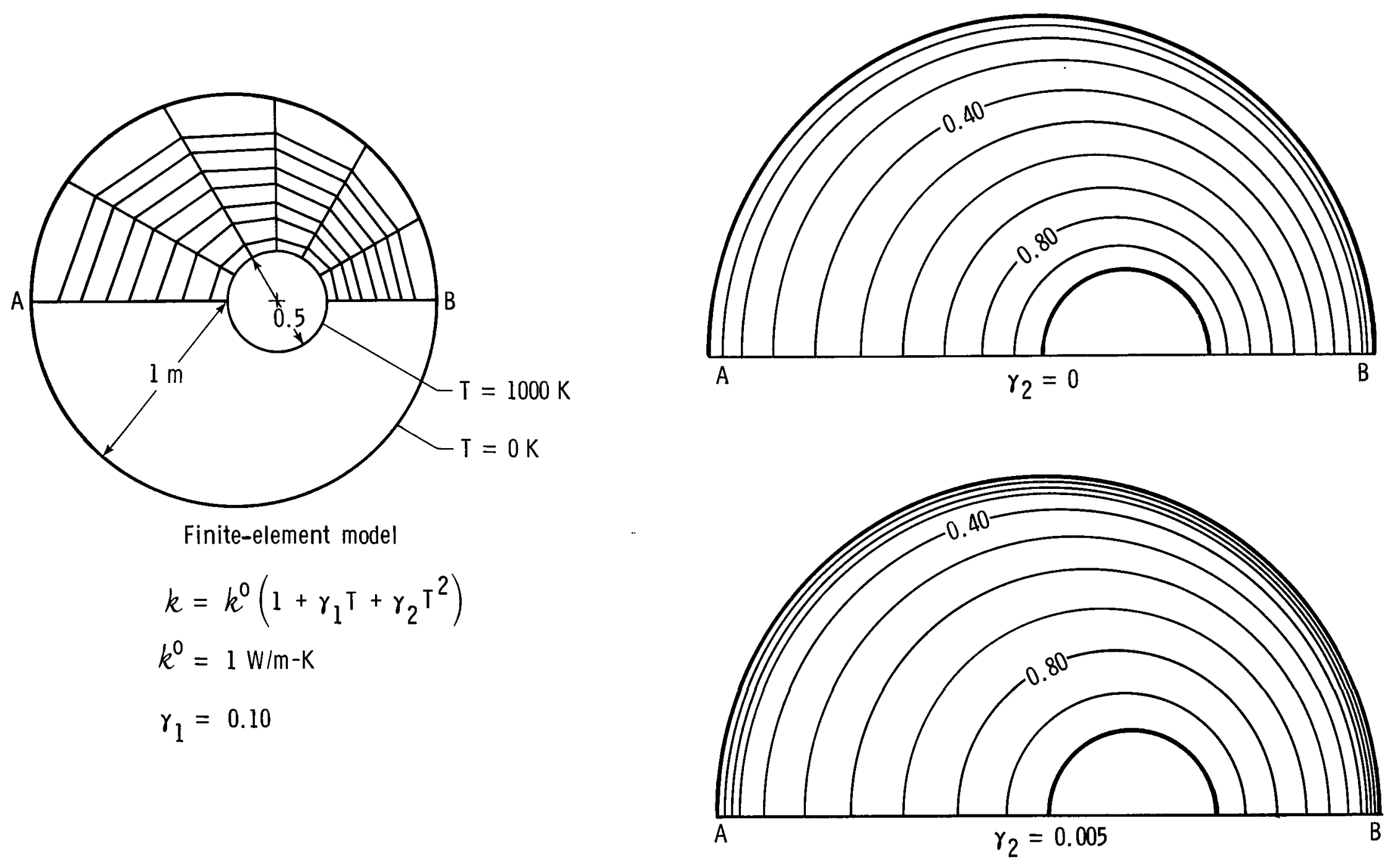

Figure 6.- Finite-element model and normalized contour plots for temperatures corresponding to $\gamma_{2}=0$ and 0.005 in a cylinder with an eccentric hole and temperature-dependent thermal conductivities. 


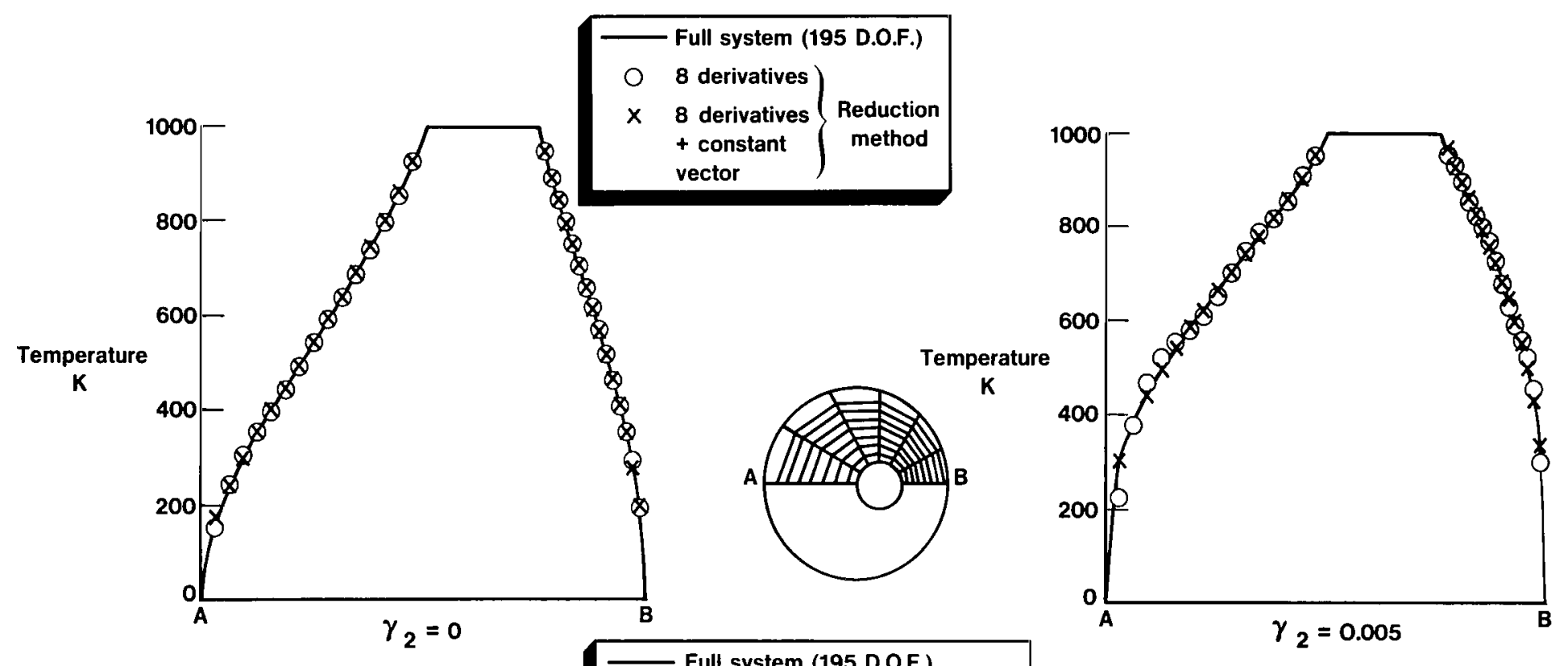

Figure 7.- Accuracy of solutions obtained by using different sets of basis vectors for cylinder having an eccentric hole and temperature-dependent thermal conductivities. 


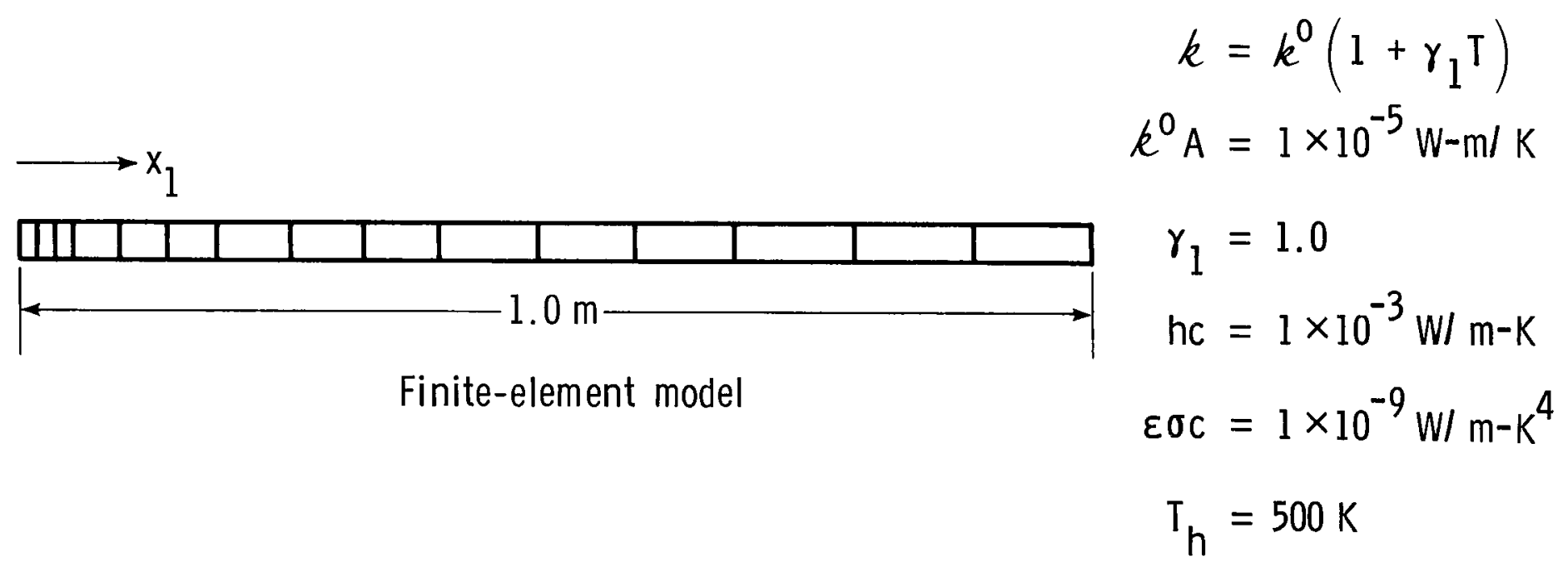

Figure 8.- Conducting-convecting-radiating fin used in the present study. $\mathrm{T}=1000 \mathrm{~K}$ at $\mathrm{x}_{1}=0$. 


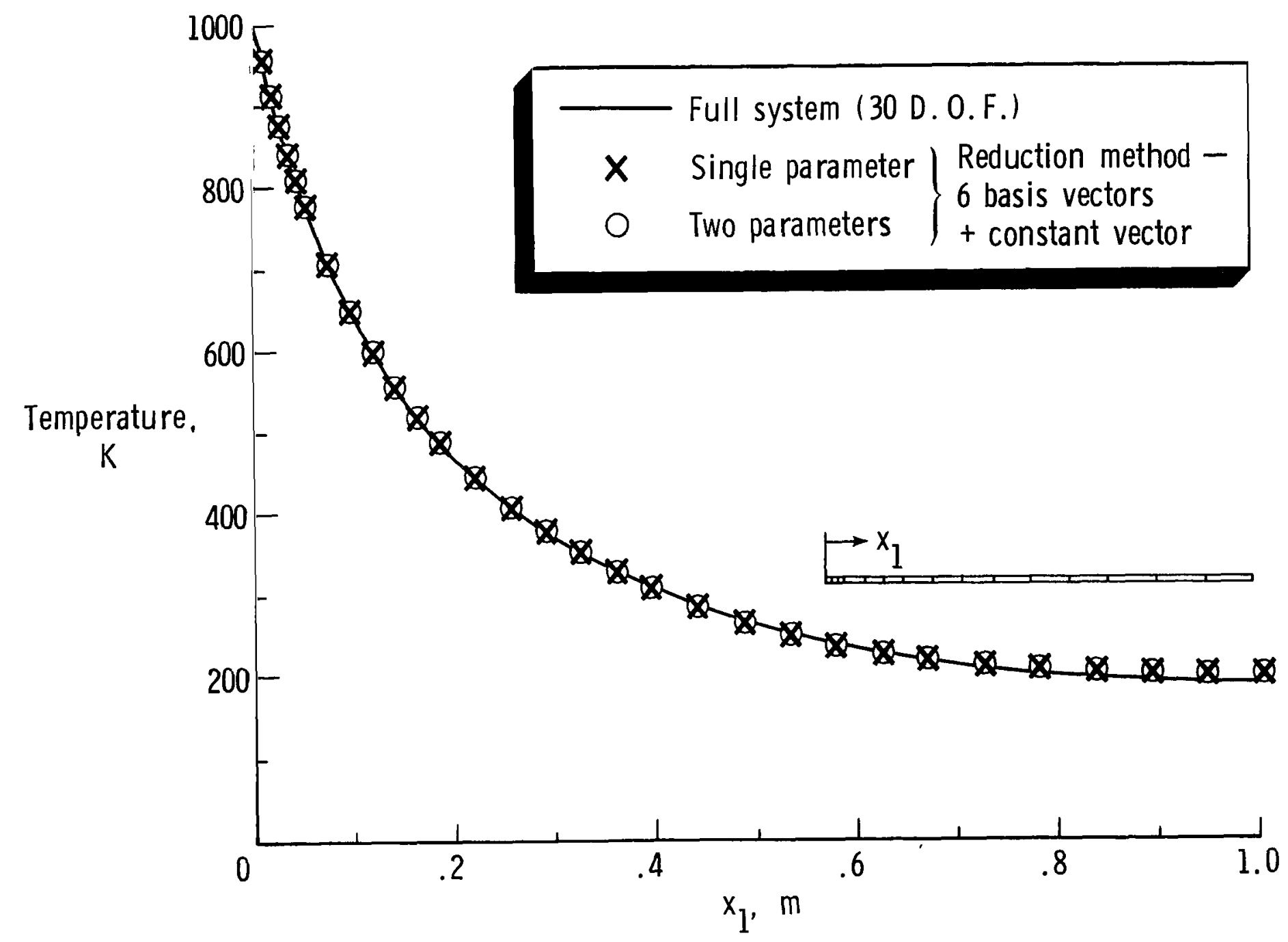

Figure 9.- Accuracy of solutions obtained by using single-parameter and two-parameter reduction methods for conducting-convecting-radiating fin. 

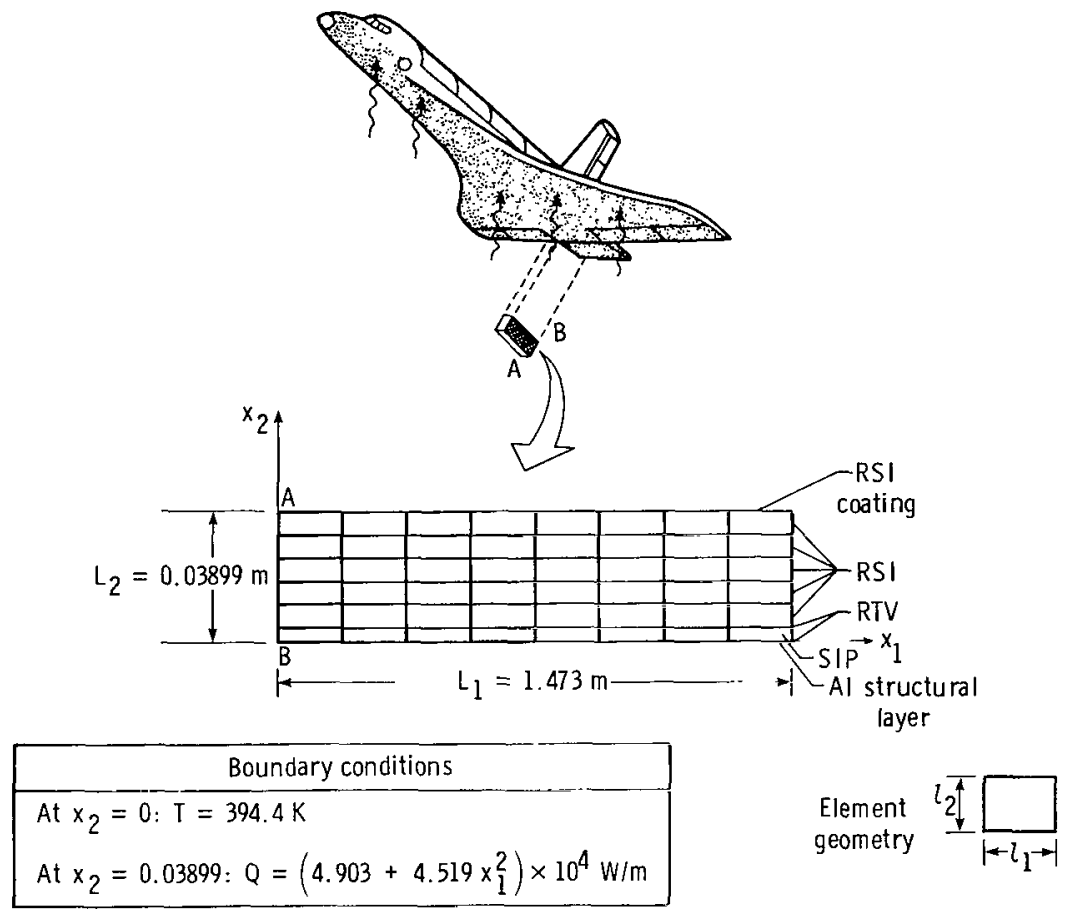

\begin{tabular}{|c|l|l|l|r|r|}
\hline \multirow{2}{*}{ Material } & \multicolumn{3}{|c|}{ Conductivity coefficients } & \multicolumn{2}{c|}{ Element dimensions } \\
\cline { 2 - 6 } & $k^{0}, \mathrm{WI} \mathrm{m-K}$ & \multicolumn{1}{c|}{$\gamma_{1}$} & $\gamma_{2}$ & $l_{1}, \mathrm{~m}$ & $l_{2}, \mathrm{~m}$ \\
\hline RSI coating & 0.5209 & $1.979 \times 10^{-3}$ & $-4.316 \times 10^{-7}$ & 0.1842 & $*$ \\
RSI & $3.974 \times 10^{-2}$ & $-9.927 \times 10^{-4}$ & $2.431 \times 10^{-6}$ & .1842 & $6.909 \times 10^{-3}$ \\
RTV & .3113 & 0 & 0 & .1842 & $1.905 \times 10^{-4}$ \\
SIP & $1.748 \times 10^{-2}$ & $1.229 \times 10^{-3}$ & $5.835 \times 10^{-6}$ & .1842 & $4.064 \times 10^{-3}$ \\
AI & $1.379 \times 10^{2}$ & 0 & 0 & .1842 & $*$ \\
\hline
\end{tabular}

*One-dimensional elements

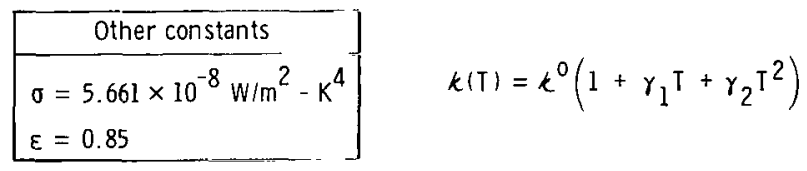

Figure 10.- Segment of the Shuttle wing bay used in the present study. 


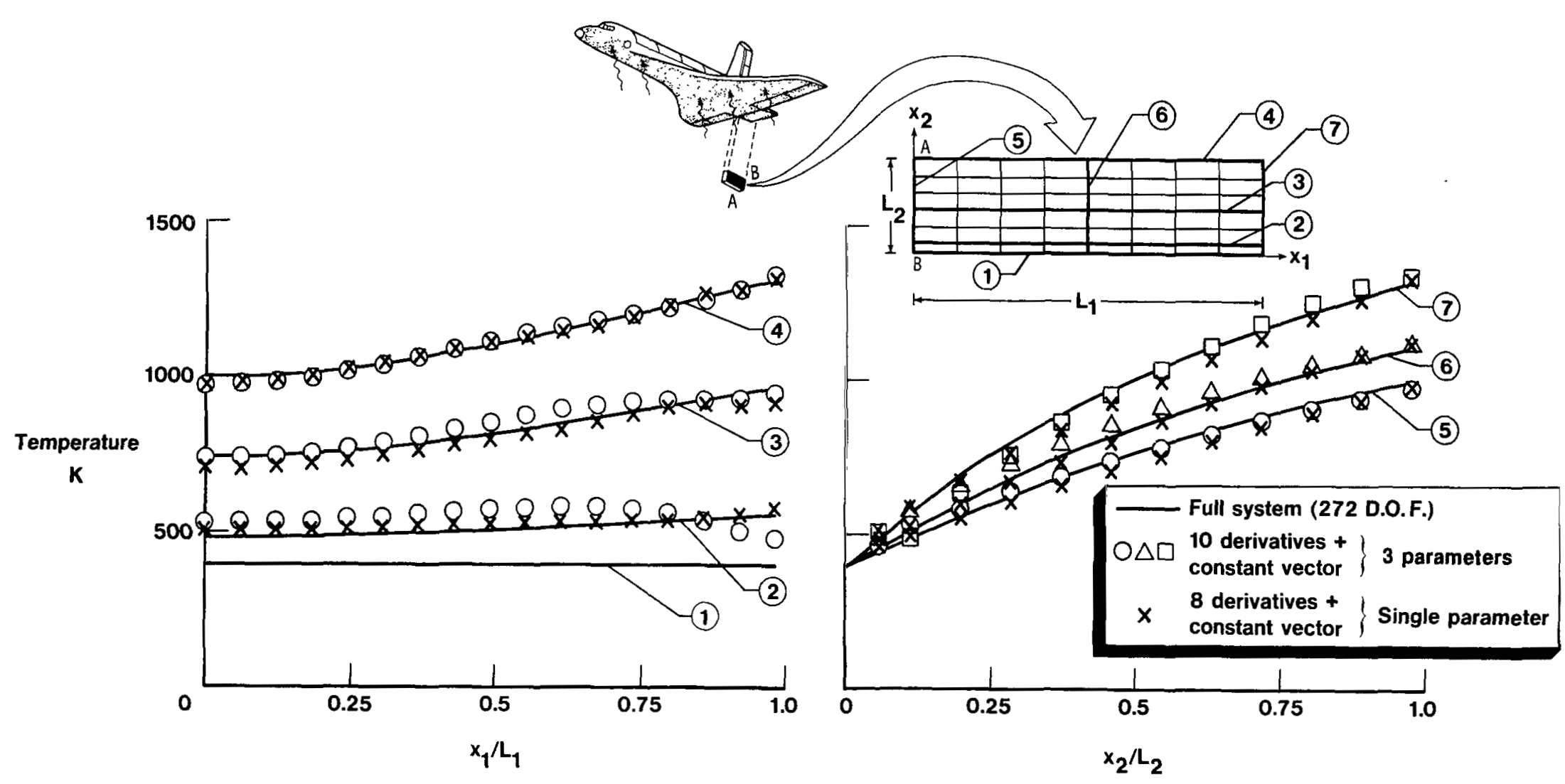

Figure 11.- Accuracy of solutions obtained by using single-parameter and three-parameter reduction methods for segment of the Shuttle wing bay. 


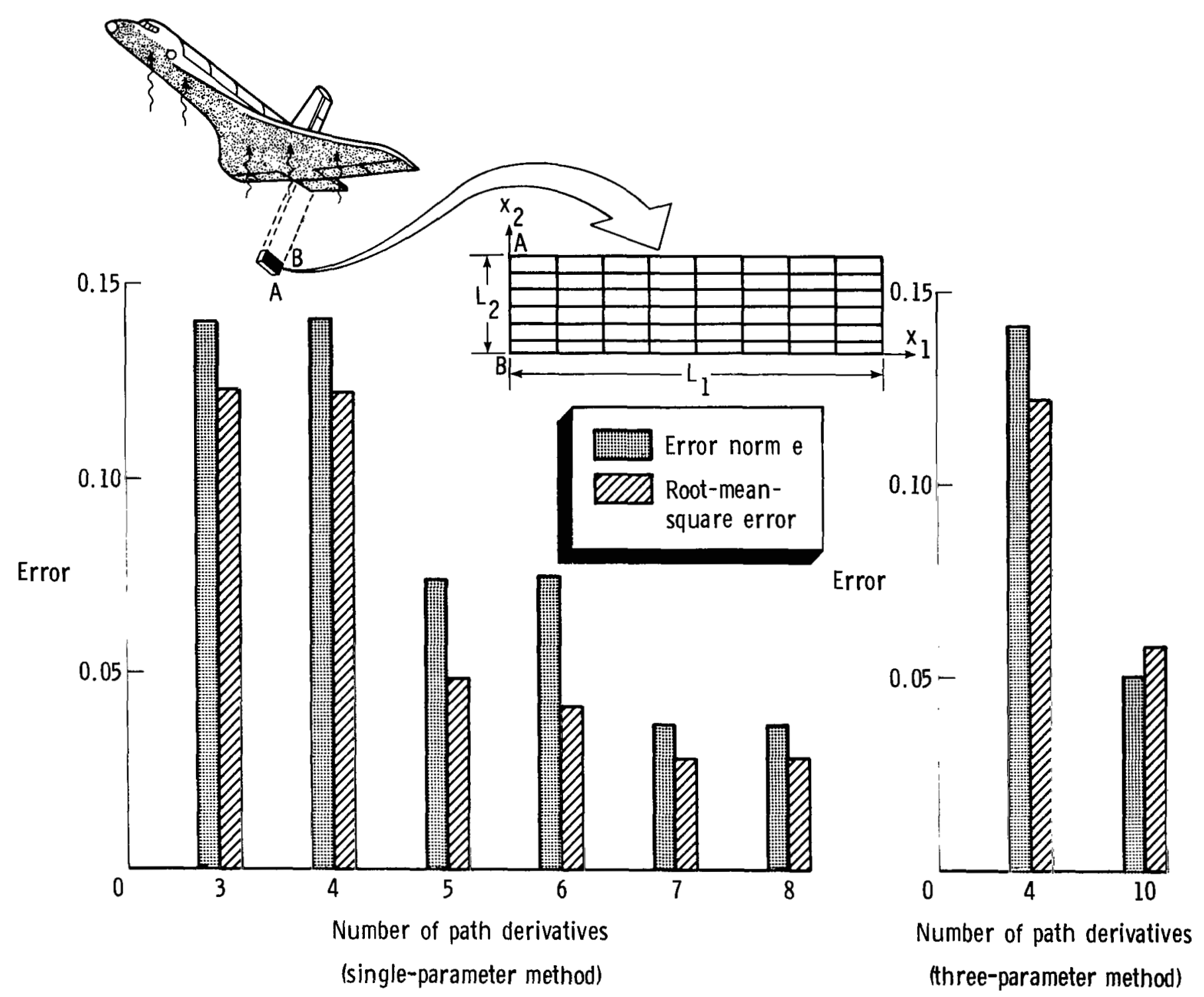

Figure 12.- Accuracy and convergence of solutions obtained by single-parameter and three-parameter reduction methods (including the constant vector) for segment of the Shuttle wing bay. 


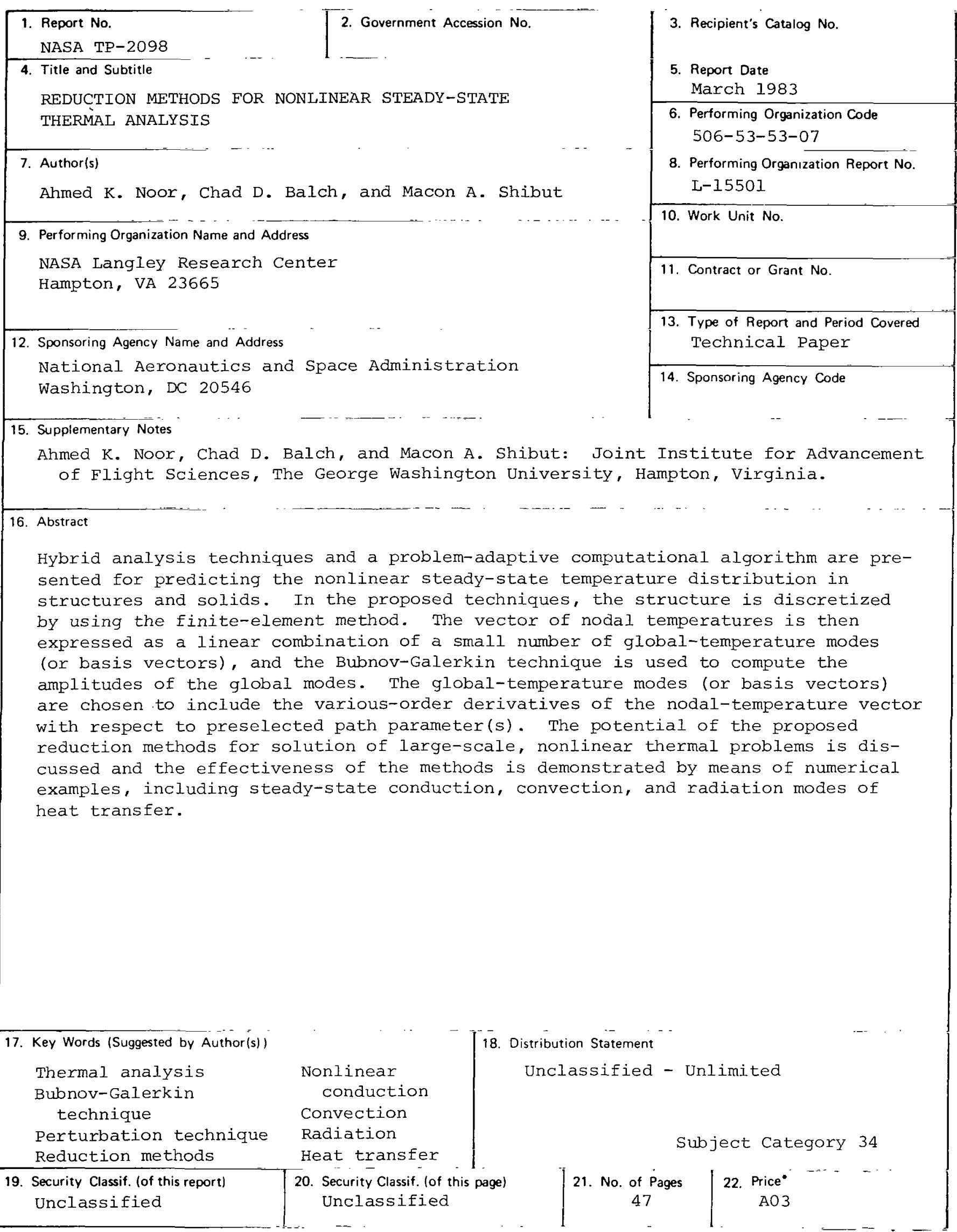

* For sale by the National Technical Information Service. Springfield, Virgınıa $22161 \quad$ NASA-Langley, 1983 
National Aeronautics and Space Administration

Washington, D.C.

20546

Official Business

Penalty for Private Use, $\$ 300$
THIRD-CLASS BULK RATE

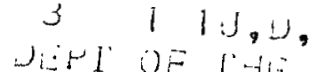

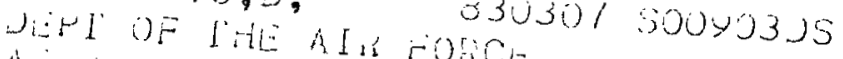



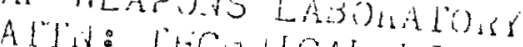

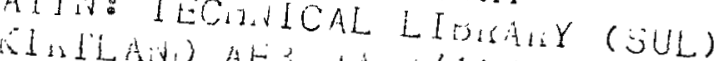

Postage and Fees Paid National Aeronautics and Space Administration NASA-451

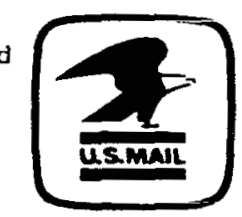

